TRANSACTIONS OF THE

AMERICAN MATHEMATICAL SOCIETY

Volume 361, Number 11, November 2009, Pages 5921-5945

S 0002-9947(09)04885-5

Article electronically published on June 15, 2009

\title{
GK-DIMENSION OF BIRATIONALLY COMMUTATIVE SURFACES
}

\author{
D. ROGALSKI
}

\begin{abstract}
Let $k$ be an algebraically closed field, let $K / k$ be a finitely generated field extension of transcendence degree 2, let $\sigma \in \operatorname{Aut}_{k}(K)$, and let $A \subseteq Q=K[t ; \sigma]$ be an $\mathbb{N}$-graded subalgebra with $\operatorname{dim}_{k} A_{n}<\infty$ for all $n \geq 0$. Then if $A$ is big enough in $Q$ in an appropriate sense, we prove that GK $A=3,4,5$, or $\infty$, with the exact value depending only on the geometric properties of $\sigma$. The proof uses techniques in the birational geometry of surfaces which are of independent interest.
\end{abstract}

\section{INTRODUCTION}

Throughout this paper, let $k$ be an algebraically closed field. Let $A=\bigoplus_{n \geq 0} A_{n}$ be a finitely generated $\mathbb{N}$-graded $k$-algebra which is locally finite $\left(\operatorname{dim}_{k} A_{n}<\infty\right.$ for all $n \geq 0$ ). There is a close relationship between such graded rings which are commutative and projective algebraic geometry; for example, a projective variety of dimension $n$ arises as Proj $A$ for a commutative graded domain $A$ of Krull dimension $n+1$. Speaking broadly, the subject of noncommutative projective geometry attempts to find interesting ways to generalize to noncommutative rings this correspondence between commutative graded $k$-algebras and projective $k$-schemes. The Gelfand-Kirillov (GK)-dimension is typically the most useful notion of dimension for rings in this theory, though it does not always match the geometric intuition. In any case, noncommutative $\mathbb{N}$-graded domains of GK-dimension 2, which correspond to noncommutative projective curves, have been classified in geometric terms by Artin and Stafford in $\mathrm{AS}$. The classification of noncommutative projective surfaces is an important ongoing research problem, but it is less clear precisely which rings should correspond to surfaces. For our purposes, given a locally finite $\mathbb{N}$ graded domain $A$ which has a graded quotient ring $Q(A) \cong D\left[t, t^{-1} ; \sigma\right]$, we want to consider $A$ as corresponding to a surface if the division ring $D$ has transcendence degree 2 over $k$. An important special case occurs when $D=K$ is a field, in which case we call the domain $A$ birationally commutative. The goal of this paper is to calculate the GK-dimension of birationally commutative surfaces.

Received by the editors September 19, 2007.

2000 Mathematics Subject Classification. Primary 14A22, 14E05, 16P90, 16S38, 16W50.

Key words and phrases. GK-dimension, graded rings, noncommutative projective geometry, noncommutative surfaces, birational geometry.

The author was partially supported by the NSF through grants DMS-0202479 and DMS0600834.

(C)2009 American Mathematical Society Reverts to public domain 28 years from publication 
The main tools to be used in the calculation are the theory of commutative projective surfaces and the Riemann-Roch theorem. There is a major difficulty to overcome: automorphisms of fields of transcendence degree 2 may not correspond nicely to automorphisms of projective surfaces. More specifically, given a finitely generated field extension $K / k$ and an automorphism $\sigma \in \operatorname{Aut}_{k}(K)$, we say that $\sigma$ is geometric if there exists a projective variety $X$ with $k(X)=K$ and an automorphism $\tau: X \rightarrow X$ which induces by pullback of rational functions the automorphism $\sigma$. Although if tr. $\operatorname{deg} K / k=1$ then every automorphism of $K$ is geometric, this sometimes fails for fields of higher transcendence degree. The main thrust of this article is to develop techniques to deal with nongeometric automorphisms.

We are now ready to state our main result. To finesse the minor technicality that graded quotient rings do not always exist, we consider more generally the GKdimension of all locally finite $\mathbb{N}$-graded subalgebras $A \subseteq K\left[t, t^{-1} ; \sigma\right]$ which are big in an appropriate sense (see Definition 6.1).

Theorem 1.1. Let $k$ be algebraically closed, let $K / k$ be a finitely generated field extension with tr. $\operatorname{deg} K / k=2$, and let $\sigma \in \operatorname{Aut}_{k}(K)$. Then every big locally finite $\mathbb{N}$-graded subalgebra $A$ of $Q=K\left[t, t^{-1} ; \sigma\right]$ has the same GK-dimension $d \in$ $\{3,4,5, \infty\}$. Moreover, if $d<\infty$, then $d=4$ if and only if $\sigma$ is not geometric, and in case $d=\infty$, then $A$ has exponential growth.

In fact, the value of GK $A$ in Theorem 1.1 is determined by geometric data associated to the automorphism $\sigma$; see Theorem 7.1 below for a more specific statement.

Given a projective $k$-scheme $X$, an automorphism $\sigma: X \rightarrow X$ and an invertible sheaf $\mathcal{L}$ on $X$, the twisted homogeneous coordinate ring $B=B(X, \mathcal{L}, \sigma)$ is the ring $\bigoplus_{n \geq 0} \mathrm{H}^{0}\left(X, \mathcal{L}_{n}\right)$, where $\mathcal{L}_{n}$ is defined by $\mathcal{L} \otimes \sigma^{*}(\mathcal{L}) \otimes \cdots \otimes\left(\sigma^{n-1}\right)^{*} \mathcal{L}$, and for $x \in B_{m}, y \in B_{n}$, the multiplication is defined by $x \star y=x \otimes\left(\sigma^{m}\right)^{*}(y)$. Such rings have good properties (such as the Noetherian property) when $\mathcal{L}$ satisfies an additional condition called $\sigma$-ampleness. For geometric automorphisms $\sigma \in \operatorname{Aut}_{k}(K)$, Theorem 1.1 is already known by the work of Zhang and the author RZ, Theorem 1.6]. The method is as follows: choose $X$ projective with $k(X)=K$ and with an automorphism $\sigma: X \rightarrow X$ corresponding to $\sigma \in \operatorname{Aut}(K)$. Then for any big subalgebra $A \subseteq Q=K\left[t, t^{-1} ; \sigma\right]$ one may show that $A$ is both contained in and contains a twisted homogeneous coordinate $\operatorname{ring} B$. In this way one obtains GK $A=\operatorname{GK} B(X, \mathcal{L}, \sigma)$ for an appropriate choice of $(\sigma$-ample $) \mathcal{L}$. Moreover, Artin and Van den Bergh showed that for a surface $X$ and $\sigma$-ample sheaf $\mathcal{L}$, the value of GK $B(X, \mathcal{L}, \sigma)$ is either 3,5 or $\infty$ [AV, Theorem 1.7]. In fact, for higher-dimensional $X$, Keeler gave bounds on the possible values of GK $B(X, \mathcal{L}, \sigma)$ Ke1, Theorem 6.1], but less is known about what values are actually obtained.

For a nongeometric $\sigma \in \mathrm{Aut}_{k} K$, we cannot pick a nice model $X$ with corresponding automorphism as in the last paragraph, so we make do by carefully picking some projective nonsingular surface $X$ with $k(X)=K$, and working with the birational map $\sigma: X \rightarrow X$ corresponding to $\sigma \in \operatorname{Aut}(K)$. Fortunately, there is recent work by Diller and Favre [DF] on the dynamics of birational self-maps of smooth surfaces over $\mathbb{C}$, which contains many helpful ideas. If $N^{1}(X)=\operatorname{Pic} X / \sim$ is the group of divisors on $X$ modulo numerical equivalence, then there is a natural way to define an action $\sigma^{*}: \operatorname{Pic}(X) \rightarrow \operatorname{Pic}(X)$ which is given roughly by pullback of divisors, and which descends to an action $\sigma^{*}: N^{1}(X) \rightarrow N^{1}(X)$. In fact $N^{1}(X) \cong \mathbb{Z}^{m}$ for some $m$ and $\sigma^{*}$ is given by some (possibly noninvertible) integer matrix $P$. As is the case 
in $\mathrm{AV}$ and $\mathrm{Ke}$ ], this matrix is the key to our GK-dimension calculations. However, the notion of pullback by a birational map is not always well-behaved under iteration; importantly, one can choose the nonsingular model $X$ of $K$ so that the birational map $\sigma: X \rightarrow X$ is also stable, in the sense that the pullback map $\left(\sigma^{n}\right)^{*}$ is given by the matrix $P^{n}$ for all $n \geq 1$ [DF, Theorem 0.1]. The cases appearing in Theorem 1.1 then correspond to the possible Jordan forms of the matrix $P$, which are classified in $\mathrm{DF}$ ] (see Theorem 3.2 below).

Since the paper $[\mathrm{DF}]$ is so fundamental to our study here, we give a rather thorough review in $\$ 2$ of those results from that paper that we need. Since the authors of $[\mathrm{DF}]$ work exclusively over $\mathbb{C}$ and use analytic methods and terminology, one of our aims here is to notice that many of their proofs use only standard algebraic surface theory, which is valid for any algebraically closed field. In a few cases where a proof in $[\mathrm{DF}$ is highly analytic, we offer an alternative algebraic proof. One of the useful aspects of the $\overline{\mathrm{DF}}]$ theory is that it shows that nongeometric automorphisms $\sigma: K \rightarrow K$ of fields $K$ of transcendence degree 2 are rather constrained: for some model $X$ of $K$, the corresponding birational map $\sigma: X \rightarrow X$ either preserves a rational fibration of $X$, or else the induced action $P: N^{1}(X) \rightarrow N^{1}(X)$ has an eigenvalue of modulus greater than 1 . We offer some observations of our own concerning the property of geometricity in $\$ 3$.

Given a surface $X$ with a stable birational map $\sigma: X \rightarrow X$ and invertible sheaf $\mathcal{L}$, we can set $\mathcal{L}_{n}=\mathcal{L} \otimes \sigma^{*} \mathcal{L} \otimes \cdots \otimes\left(\sigma^{n-1}\right)^{*} \mathcal{L}$ and build an analog of a twisted homogeneous coordinate ring, which we write as $\widetilde{B}(X, \mathcal{L}, \sigma)=\bigoplus_{n \geq 0} \mathrm{H}^{0}\left(X, \mathcal{L}_{n}\right)$ and call a twisted section ring. This construction reduces to the usual twisted homogeneous coordinate ring when $\sigma$ is an automorphism. We give the construction of these rings in 84 Unfortunately, for nongeometric $\sigma$ such rings are badly behaved: if $\mathcal{L}$ is also ample enough in some sense, then we show that $\widetilde{B}(X, \mathcal{L}, \sigma)$ is not finitely generated as an algebra. However, we can still calculate the growth of the graded pieces of $\widetilde{B}$ using the Riemann-Roch theorem, and we do this calculation in $\$ 5$, Given a finitely generated big subalgebra $A$ of $K\left[t, t^{-1} ; \sigma\right]$, it is easy to show that $A$ is contained in some $\widetilde{B}$, which gives an upper bound on GK $A$. Finding a lower bound on GK $A$ is trickier, though, since in general we cannot find a copy of some $\widetilde{B}$ as a subalgebra of $A$. For the lower bound, we restrict all sections to some curve $E$ on $X$ and reduce the problem to some growth estimates for divisors on the curve $E$. The details are given in $\$ 6$ Then the upper and lower bound estimates are combined to prove the main theorem in 97

The main theorem has applications to the classification theory of noncommutative projective surfaces. In [RS1, Theorem 1.1], we completely described $\mathbb{N}$ graded domains $A$ which are Noetherian, generated in degree 1 , and have $Q(A)=$ $K\left[t, t^{-1} ; \sigma\right]$ for a finitely generated field extension $K$ with tr. $\operatorname{deg} K / k=2$ and $\sigma$ geometric. Namely, in large degrees such an $A$ is isomorphic either to a twisted homogeneous coordinate ring $B(X, \mathcal{L}, \sigma)$, or to a naïve blow-up algebra $R(X, Z, \mathcal{L}, \sigma)$ as studied in [KRS] and [RS2]. For a nongeometric $\sigma$, what finitely generated domains $A$ with $Q(A)=K\left[t, t^{-1} ; \sigma\right]$ might look like is still unclear; we don't know, for example, if any such $A$ are Noetherian. Indeed, we have developed here the background material about nongeometric automorphisms and twisted section rings in greater detail than we really need for the proof of the main theorem about GKdimension, because we hope this will be of use in further work to better understand such domains $A$. In any case, Theorem 1.1 gives us a first understanding of what the 
Noetherian examples (if any) for nongeometric $\sigma$ look like: they have GK-dimension 4 (since Noetherian $\mathbb{N}$-graded algebras cannot have exponential growth). Additionally, Theorem 1.1 allows the hypothesis that $\sigma$ is geometric in [RS1, Theorem 1.1] to be replaced with the alternative hypothesis that GK $A=3$ or 5 .

\section{Review of Results of Diller And FAVRe}

In this section, the word surface will always mean a nonsingular integral projective surface over the algebraically closed field $k$. The letters $W, X, Y, Z$ will always stand for surfaces. On such a surface $X$, we can identify Pic $X$ with the set of Weil divisors $D$ up to linear equivalence or with invertible sheaves up to isomorphism. There is a symmetric nondegenerate bilinear intersection form $(\cdot, \cdot)$ on Pic $X$, as defined in [Ha, Section V.1]. We say that divisors $D, E$ are numerically equivalent if $(D-E . C)=0$ for all divisors $C$. The quotient of $\operatorname{Pic} X$ by the relation of numerical equivalence is the Neron-Severi group $N^{1}(X)=\operatorname{Pic} X / \sim$, which is a free abelian group of finite rank. A divisor $D$ is nef if $(D . C) \geq 0$ for all irreducible curves $C$ on $X$. We also work with $\mathbb{R}$-divisors up to numerical equivalence, that is, the group $N^{1}(X)_{\mathbb{R}}=N^{1}(X) \otimes_{\mathbb{Z}} \mathbb{R}$; the intersection form uniquely extends to a form on $N^{1}(X)_{\mathbb{R}}$, and $D \in N^{1}(X)_{\mathbb{R}}$ is called nef if $(D . C) \geq 0$ for all irreducible curves $C$ on $X$. The set of nef classes forms the nef cone $\operatorname{Nef}(X) \subseteq N^{1}(X)_{\mathbb{R}}$. The pseudoeffective cone $\overline{\mathrm{NE}}(X) \subseteq N^{1}(X)_{\mathbb{R}}$ is the smallest closed cone in $N^{1}(X)_{\mathbb{R}}$ containing the classes of all effective curves. The nef cone and pseudoeffective cone are dual with respect to the intersection form, and a nef class is also pseudoeffective. See [La] for more information on all of these standard facts.

We now review the notion of pullback of divisors by a birational map.

Definition 2.1. Let $f: X \rightarrow Y$ be a birational map of surfaces. The map $f$ is defined except at a finite set of fundamental points. Let $S \subseteq X$ be any finite set of points in $X$ containing all of the fundamental points of $f$, and let $U=X \backslash S$, so $\left.f\right|_{U}: U \rightarrow Y$ is a morphism. Then given any divisor $D$ on $Y$, the divisor $\left(\left.f\right|_{U}\right)^{*}(D)$ on $U$ extends uniquely to a divisor $f^{*}(D)$ on $X$. In this way, we define a map $f^{*}: \operatorname{Pic} Y \rightarrow \operatorname{Pic} X$.

In the proofs below, it is useful to have a more explicit definition of the pullback map $f^{*}$ which reduces to the case of a monoidal transformation, and we discuss this next. Suppose first that $\rho: W \rightarrow X$ is a birational surjective morphism. Then $\rho=\rho_{1} \rho_{2} \ldots \rho_{n}$ is a composition of finitely many monoidal transformations $\rho_{i}: W_{i} \rightarrow W_{i-1}$ for some surfaces $W_{0}=X, W_{1}, \ldots, W_{n}=W$ [Ha, Corollary V.5.4]. If $E \subseteq W_{i}$ is the exceptional curve for $\rho_{i}$, then set $F_{i}=\left(\rho_{i+1} \rho_{i+2} \ldots \rho_{n}\right)^{*}(E)$. It then follows from [Ha, Propositions V.3.2, V.3.6] that the pullback map $\rho^{*}$ : Pic $X \rightarrow \operatorname{Pic} W$ is an injection, with

$$
\text { Pic } W=\rho^{*}(\operatorname{Pic} X) \oplus \mathbb{Z} F_{1} \oplus \cdots \oplus \mathbb{Z} F_{n},
$$

where $\left(F_{i} \cdot F_{i}\right)=-1$ for all $i,\left(F_{i} \cdot F_{j}\right)=0$ for $i \neq j,\left(D \cdot F_{i}\right)=0$ for all $D \in \rho^{*}(\operatorname{Pic} X)$ and all $i$, and $\left(\rho^{*} C_{1}, \rho^{*} C_{2}\right)_{W}=\left(C_{1} . C_{2}\right)_{X}$ for all $C_{1}, C_{2} \in \operatorname{Pic} X$. Moreover, each $F_{i}$ is a nonnegative linear combination of the finitely many irreducible curves contracting under $\rho$ and so is effective. Given any irreducible curve $C \subseteq X$, if $\widetilde{C} \subseteq W$ is the proper transform of $C$, then iterating [Ha, Proposition V.3.6] we have $\rho^{*}(C)=\widetilde{C}+\sum a_{i} F_{i}$ for some nonnegative $a_{i}$. We can also push forward divisors: $\rho_{*}$ : Pic $W \rightarrow \operatorname{Pic} X$ simply projects onto the factor $\rho^{*}(\operatorname{Pic} X) \cong \operatorname{Pic} X$. 
Alternatively, it is clear that given an irreducible curve $D \subseteq Y$, then $\rho_{*}(D)=\rho(D)$ if $\rho(D)$ is a curve or 0 if $\rho(D)$ is a point, and extending this rule linearly determines the map $\rho_{*}$.

Now if $f: X \rightarrow Y$ is a birational map, then there exists another surface $W$ and birational morphisms $\phi: W \rightarrow X, \psi: W \rightarrow Y$ such that $f=\psi \phi^{-1}$ Ha, Theorem V.5.5]. Then it is easy to check that $f^{*}=\phi_{*} \psi^{*}: \operatorname{Pic} Y \rightarrow \operatorname{Pic} X$ is the same map as defined in Definition 2.1 in particular, this is independent of the choice of $W$. We also define the pushforward of divisors by $f$ by setting $f_{*}=\left(f^{-1}\right)^{*}=\psi_{*} \phi^{*}$ : Pic $X \rightarrow \operatorname{Pic} Y$. The pullback map $f^{*}$ respects numerical equivalence, and so it also induces maps $f^{*}: N^{1}(Y) \rightarrow N^{1}(X)$ and $f^{*}: N^{1}(Y)_{\mathbb{R}} \rightarrow N^{1}(X)_{\mathbb{R}}$.

In the paper [DF, Diller and Favre define the pullback of cohomology classes by a birational map $f: X \rightarrow Y$ in case $k=\mathbb{C}$, using the language of complex geometry [DF, Definition 1.8]. They then give a very useful and beautiful classification of birational self-maps of surfaces. Our main aim in this section is to review their theory and to show that a large number of their results holds over any algebraically closed field. For most of their proofs, we simply checked that upon substituting the algebraic language of divisors for their more analytic language, the same proof works. In this case, we simply state their result below without proof. In a few cases where the translation of their proof to the algebraic setting is nonobvious, or where we need a slightly stronger formulation of a result, we provide a proof here. We review not only the main results from $[\overline{D F}$ that we need in the sequel, but also the subsidiary results on which they depend. We hope this will help the careful reader who wants to check that everything goes through over arbitrary algebraically closed fields, as well as give all readers a better idea of the flavor of the theory.

We begin with several results which follow very formally from the definitions of pullback and pushforward.

Lemma 2.2 ([DF, Proposition 1.11(2)(3)]). Let $f: X \rightarrow Y$ be a birational map. Then

(1) (Adjointness) $\left(f^{*} C . D\right)_{X}=\left(C . f_{*} D\right)_{Y}$ for all $C \in N^{1}(Y)_{\mathbb{R}}, D \in N^{1}(X)_{\mathbb{R}}$.

(2) The map $f^{*}: N^{1}(Y)_{\mathbb{R}} \rightarrow N^{1}(X)_{\mathbb{R}}$ preserves the nef and pseudoeffective cones; in other words, $f^{*}(\operatorname{Nef}(Y)) \subseteq \operatorname{Nef}(X)$ and $f^{*}(\overline{\mathrm{NE}}(Y)) \subseteq \overline{\mathrm{NE}}(X)$.

Pullback by a birational map does not generally respect the intersection form. The next result calculates the exact nature of the discrepancy.

Lemma 2.3 ([DF, Corollary 3.4]). Let $f: X \rightarrow Y$ be a birational map and choose another surface $W$ and birational morphisms $\phi: W \rightarrow X, \psi: W \rightarrow Y$ such that $f=\psi \phi^{-1}$. Decompose $\operatorname{Pic} W \cong \phi^{*}(\operatorname{Pic} X) \oplus \mathbb{Z} F_{1} \oplus \cdots \oplus \mathbb{Z} F_{n}$ with respect to the morphism $\phi$, as in (2.1), and let $E_{i}=\psi_{*}\left(F_{i}\right) \in \operatorname{Pic} Y$. Then each $E_{i}$ is a sum with nonnegative coefficients of irreducible curves contracting under $f^{-1}$. Moreover:

(1) $\left(f^{*}(C) . f^{*}(D)\right)=(C . D)+\sum_{i=1}^{n}\left(C . E_{i}\right)\left(D . E_{i}\right)$ for all $C, D \in N^{1}(Y)_{\mathbb{R}}$.

(2) $\left(f^{*}(D) \cdot f^{*}(D)\right)=(D . D)$ for some $D \in N^{1}(Y)_{\mathbb{R}}$ if and only if $\left(D . V_{i}\right)=0$ for all irreducible curves $V_{i} \subseteq Y$ contracted by $f^{-1}$.

Proof. The proof of [DF, Corollary 3.4], which needs no essential change, depends only on the earlier results [DF, Proposition 3.1, Proposition 3.2, Theorem 3.3]. These results also go through with only obvious changes. In fact, the algebraic versions of $\mathrm{DF}$, Proposition 3.1 and Proposition 3.2] are immediate from [Ha, Proposition V.3.6 and Corollary V.3.7] (note that we assume $f$ is birational whereas 
$f$ is allowed to be any surjective morphism in these results in $[\mathrm{DF}$; in particular, the constant $\lambda_{2}$ of [DF, Proposition 3.1] satisfies $\lambda_{2}=1$ in our setting).

The behavior of the pullback maps under composition also needs careful examination.

Lemma 2.4 ([DF, Proposition 1.13]). Suppose that $f: X \rightarrow Y$ and $g: Y \rightarrow Z$ are birational maps, and consider $f^{*}: \operatorname{Pic} Y \rightarrow \operatorname{Pic} X, g^{*}: \operatorname{Pic} Z \rightarrow \operatorname{Pic} Y$, and $(g f)^{*}: \operatorname{Pic} Z \rightarrow \operatorname{Pic} X$.

(1) For any nef divisor $D \in \operatorname{Pic} Z, f^{*} g^{*}(D)-(g f)^{*}(D)$ is effective.

(2) If there does not exist any curve $C \subseteq X$ such that $f(C)=p$ is a fundamental point of $g$, then $f^{*} g^{*}=(g f)^{*}$.

Proof. Since the proof in DF seems quite analytic, we provide an algebraic proof here. We may find a surface $W_{1}$ and birational morphisms $\phi: W_{1} \rightarrow X, \psi: W_{1} \rightarrow Y$ such that $f=\psi \phi^{-1}$, and a surface $W_{2}$ and birational morphisms $\mu: W_{2} \rightarrow Y$, $\nu: W_{2} \rightarrow Z$ such that $g=\nu \mu^{-1}$. Then let $h=\mu^{-1} \psi: W_{1} \rightarrow W_{2}$ and find a surface $W_{3}$ and birational morphisms $\tau: W_{3} \rightarrow W_{1}, \theta: W_{3} \rightarrow W_{2}$ such that $h=\theta \tau^{-1}$.

Let $D \in \operatorname{Pic} Z$, and let $E=\nu^{*} D \in \operatorname{Pic} W_{2}$. Let $P$ be the finite set of fundamental points of $\mu^{-1}$, and let $Q=\left\{x \in W_{1} \mid \psi(x) \in P\right\}$, which is a proper closed subset of $W_{1}$. Let $G_{1}, G_{2}, \ldots, G_{m} \subseteq W_{1}$ be the distinct irreducible curves contained in $Q$. Let $T=W_{1} \backslash Q$ and $U=Y \backslash P$. Then the birational map $\mu^{-1} \psi$ is represented by the morphism $\left(\left.\mu^{-1}\right|_{U}\right) \circ\left(\left.\psi\right|_{T}\right): T \rightarrow W_{2}$. Similarly, let $R \subseteq W_{1}$ be the finite set of fundamental points of $\tau^{-1}$ and put $V=W_{1} \backslash R$, so the birational map $\theta \tau^{-1}$ is represented by the morphism $\theta \circ\left(\left.\tau^{-1}\right|_{V}\right): V \rightarrow W_{2}$. Since $\mu^{-1} \psi=\theta \tau^{-1}$ as birational maps, it now follows from Definition 2.1 that $\psi^{*} \mu_{*} E-\tau_{*} \theta^{*} E$ must be supported along the curves $G_{1}, \ldots, G_{m}$, i.e. $\psi^{*} \mu_{*} E-\tau_{*} \theta^{*} E=\sum_{i=1}^{m} a_{i} G_{i} \in \operatorname{Pic} W_{1}$. Then $f^{*} g^{*}=\phi_{*} \psi^{*} \mu_{*} \nu^{*}$ and $(g f)^{*}=\phi_{*} \tau_{*} \theta^{*} \nu^{*}$ and so $f^{*} g^{*}(D)-(g f)^{*}(D)=$ $\sum_{i=1}^{m} a_{i} \phi_{*}\left(G_{i}\right)$. In particular, in the case of part (2), the set of $G_{i}$ 's is empty and so the equation $f^{*} g^{*}=(g f)^{*}$ follows.

Now suppose that $D$ is nef as in part (1). Then $E=\nu^{*} D$ is also nef by Lemma 2.2(2). Write Pic $W_{1}=\psi^{*}(\operatorname{Pic} Y) \oplus \mathbb{Z} F_{1} \oplus \cdots \oplus \mathbb{Z} F_{n}$ with respect to the morphism $\psi$ as in (2.1). Since $\psi^{*} \mu_{*} E \in \psi^{*}(\operatorname{Pic} Y),\left(\psi^{*} \mu_{*} E . F_{i}\right)=0$ for all $i$. Because $\tau_{*} \theta^{*} E$ is also nef by Lemma $2.2(2)$ and $F_{i}$ is effective, $\left(\tau_{*} \theta^{*} E . F_{i}\right) \geq 0$ for each $i$. Setting $B=\psi^{*} \mu_{*} E-\tau_{*} \theta^{*} E$, we have $\left(B . F_{i}\right) \leq 0$ for all $i$. Since each $G_{i}$ contracts under $\psi, G_{i} \in \sum \mathbb{Z} F_{i}$. Also, by the first part of the proof, we have $B \in \sum \mathbb{Z} G_{i}$. Thus $B=\sum_{i=1}^{n} b_{i} F_{i}$. Since $\left(F_{i} \cdot F_{j}\right)=-\delta_{i j}$, this forces $b_{i} \geq 0$ for all $i$ and so $B$ is effective. Finally, $f^{*} g^{*}(D)-(g f)^{*}(D)=\phi_{*}(B)$ is then also effective.

Now we want to concentrate on the special case of a birational map $\sigma: X \rightarrow X$ from a surface $X$ to itself. In particular, we want to study the pullback map $\sigma^{*}: N^{1}(X)_{\mathbb{R}} \rightarrow N^{1}(X)_{\mathbb{R}}$. Recalling that $N^{1}(X)$ is a free Abelian group $\cong \mathbb{Z}^{d}$ for some $d$, the map $\sigma^{*}$ is given by some matrix in $M_{d}(\mathbb{Z})$.

Example 2.5. As Lemma 2.4 suggests, the pullback map $\sigma^{*}$ may behave unpredictably with respect to iteration. For example, suppose that $X=\mathbb{P}^{2}$ and $\sigma: \mathbb{P}^{2} \rightarrow \mathbb{P}^{2}$ is the Cremona map defined by $(a: b: c) \mapsto(b c: a c: b a)$. Then $\operatorname{Pic} X=N^{1}(X) \cong \mathbb{Z}$ and $\sigma^{*}: \mathbb{Z} \rightarrow \mathbb{Z}$ is multiplication by 2 . However, $\sigma^{2}$ is the identity and so $\left(\sigma^{2}\right)^{*}$ is also the identity. 
The previous example shows that we cannot expect $\left(\sigma^{n}\right)^{*}=\left(\sigma^{*}\right)^{n}$ to hold in general. The next definition singles out birational maps without this deficiency.

Definition 2.6. The birational map $\sigma: X \rightarrow X$ is called stable (the term analytically stable is used in $[\overline{\mathrm{DF}}]$ ) if there does not exist an irreducible curve $C \subseteq X$ and $n \geq 1$ such that $\sigma^{n}(C)$ is a fundamental point of $\sigma$.

For example, the Cremona map $\sigma: \mathbb{P}^{2} \rightarrow \mathbb{P}^{2}$ described above is not stable, because $\sigma$ contracts the line $\{(a: b: c) \mid a=0\}$ to the point $(1: 0: 0)$ where $\sigma$ is undefined.

Remark 2.7. It is an easy consequence of the definition, using that a point $p \in Y$ is a fundamental point for some birational map $\rho: Y \rightarrow Z$ if and only if $\rho^{-1}$ contracts some curve to $p[\mathrm{Sh}$, p. 256], that $\sigma: X \rightarrow X$ is stable if and only if $\sigma^{-1}$ is stable. Also, note that if $\sigma: X \rightarrow X$ is stable and $C$ is a curve such that $\sigma(C)=p_{0}$ is a point, then $\sigma\left(p_{0}\right)=p_{1}$ is defined; then since $\sigma^{2}(C)=p_{1}, \sigma\left(p_{1}\right)=p_{2}$ is defined, and so on. Continuing inductively, we can define a sequence of points $p_{i}$ by the rule $\sigma\left(p_{i}\right)=p_{i+1}$.

Lemma 2.8 ([DF, Theorem 1.14]). If $\sigma: X \rightarrow X$ is stable, then $\left(\sigma^{n}\right)^{*}=\left(\sigma^{*}\right)^{n}$ and $\left(\sigma^{n}\right)_{*}=\left(\sigma_{*}\right)^{n}$ for all $n \geq 1$.

Proof. This is an immediate consequence of Lemma 2.4(2) and Remark 2.7.

If $\sigma: X \rightarrow X$ is a birational map and $f: Y \rightarrow X$ is another birational map, then we say that the birational map $\tau=f^{-1} \sigma f: Y \rightarrow \rightarrow Y$ is conjugate to $\sigma$. One of the most fundamental results in $[\mathrm{DF}]$ is that any birational self-map is conjugate to a stable one.

Theorem 2.9 ([DF, Theorem 0.1]). Let $\sigma: X \rightarrow X$ be a birational map. Then there is another surface $Y$ and a birational morphism $\pi: Y \rightarrow X$ such that $\pi^{-1} \sigma \pi$ : $Y \rightarrow-Y$ is stable.

Next, for a birational map $\sigma: X \rightarrow X$ we study how the maps $\left(\sigma^{n}\right)^{*}$ : $N^{1}(X)_{\mathbb{R}} \rightarrow N^{1}(X)_{\mathbb{R}}$ grow with $n$. In this paper, it is most convenient to measure the growth of functions using a different equivalence relation than that traditionally used in the theory of GK-dimension [KL, p. 5]. Given two functions $f, g: \mathbb{N} \rightarrow \mathbb{R}$ which are monotone increasing and positive-valued for all $n \gg 0$, we write $f \preccurlyeq g$ if there is a constant $d>0$ such that $f(n) \leq d g(n)$ for all $n \gg 0$. If $f \preccurlyeq g$ and $g \preccurlyeq f$, then we write $f \sim g$. There is no difference between this definition and the one given in [KL, p. 5] for functions of polynomial growth, but our definition distinguishes between various functions of exponential growth such as $f(n)=n 2^{n}$ and $g(n)=2^{n}$. It is also useful to note that if $\lim _{n \rightarrow \infty} f(n) / g(n)$ is a finite positive number, then $f \sim g$.

For any surface $X,\|\cdot\|$ will indicate some arbitrary matrix norm on the space $\operatorname{End}_{\mathbb{C}}\left(N^{1}(X)_{\mathbb{C}}\right)$. The particular choice of matrix norm will never impact any of our results below. Recall that $\rho=\max \left\{|\lambda| \mid \lambda \in \mathbb{C}\right.$ is an eigenvalue of $\left.\sigma^{*}\right\}$ is called the spectral radius of $\sigma^{*}$.

Lemma 2.10 ([DF, Lemma 1.12]). Let $\sigma: X \rightarrow X$ be a birational map and let $\sigma^{*}: N^{1}(X)_{\mathbb{R}} \rightarrow N^{1}(X)_{\mathbb{R}}$ have spectral radius $\rho$. Then there is a nef class $D \in N^{1}(X)_{\mathbb{R}}$ which is an eigenvector for $\sigma^{*}$ with eigenvalue $\rho$. 
Proof. We note here that this result follows quickly from existing references. By Lemma 2.2, $\sigma^{*}$ preserves the nef cone $\operatorname{Nef}(X) \subseteq N^{1}(X)_{\mathbb{R}}$. Then the spectral radius $\rho$ is an eigenvalue of $\sigma^{*}$ with an eigenvector in $\operatorname{Nef}(X)$ by $[V$, Theorem 3.1]. (This is the same argument used by Keeler, for the case where $\sigma$ is an automorphism, in [Ke1, Lemma 3.2]; only the fact that $\sigma^{*}$ preserves the nef cone is needed.)

Lemma 2.11 ([DF, Corollary 1.16]). Let $\sigma: X \rightarrow X$ be a birational map.

(1) For any birational map $\tau: Y \rightarrow Y$ which is conjugate to $\sigma$, one has $\left\|\left(\sigma^{n}\right)^{*}\right\| \sim\left\|\left(\tau^{n}\right)^{*}\right\|$.

(2) $\left\|\left(\sigma^{n}\right)^{*}\right\| \sim n^{j} \rho^{n}$ for some uniquely determined real $\rho \geq 1$ and integer $j \geq 0$.

(3) If $\sigma$ is stable, then $\rho$ is the spectral radius of $\sigma^{*}$ and $j+1$ is the size of the largest Jordan block in $\sigma^{*}$ associated to an eigenvalue of modulus $\rho$.

Proof. The proof of [DF, Corollary 1.16], which depends on [DF, Proposition 1.15] and Lemma 2.4(1), goes through with little change to prove part (1). If $\sigma$ is stable, then $\left(\sigma^{n}\right)^{*}=\left(\sigma^{*}\right)^{n}$. Choosing a basis of $N^{1}(X)_{\mathbb{C}}$ so that $\sigma^{*}$ is in Jordan form, each coordinate of the matrix $\left(\sigma^{*}\right)^{n}$ is a $\mathbb{C}$-linear combination of terms of the form $n^{a} \lambda^{i}$, where $a \geq 0,0 \leq i \leq n$, and $\lambda$ is an eigenvalue of $\sigma^{*}$. If $j+1$ is the size of the largest Jordan block in $\sigma^{*}$ associated to an eigenvalue $\lambda$ of modulus $\rho$, then $n^{j} \lambda^{n}$ is the fastest growing such term; thus $\left\|\left(\sigma^{n}\right)^{*}\right\| \sim n^{j} \rho^{n}$ in this case, proving part (3). Since each $\left(\sigma^{n}\right)^{*}$ is in $\operatorname{End}_{\mathbb{Z}}\left(N^{1}(X)\right) \cong M_{d}(\mathbb{Z})$ and there is a positive lower bound on the norms of nonzero integer matrices, it follows that $\rho \geq 1$. For a $\sigma$ which is not necessarily stable, it now follows from Theorem 2.9 and part (1) that $\left\|\left(\sigma^{n}\right)^{*}\right\| \sim n^{j} \rho^{n}$ for some $\rho, j$ with $\rho \geq 1$. The values of $\rho, j$ are obviously uniquely determined given our definition of equivalence of functions, completing the proof of part (2).

Definition 2.12. Given a birational map $\sigma: X \rightarrow X$, we associate to $\sigma$ the growth data $(\rho, j)$ as determined by Lemma 2.11(2).

The main achievement of $[\mathrm{DF}$ is to classify the possible values of the growth data $(\rho, j)$ associated to birational maps $\sigma: X \rightarrow X$ and to describe which kinds of surfaces can appear in each case. The last results from $\overline{D F}$ that we recall in this section contain the main work for this classification. We need a few more definitions. First, a rational fibration of $X$ is a morphism $f: X \rightarrow C$, where $C$ is a nonsingular projective curve and the generic fiber of $f$ is isomorphic to $\mathbb{P}^{1}$. A birational map $\sigma: X \rightarrow X$ preserves such a fibration if there is an automorphism $\tau: C \rightarrow C$ such that $f \sigma=\tau f$ (as birational maps).

Lemma 2.13 ([DF, Proposition 1.7]). Let $\sigma: X \rightarrow X$ be a birational map which is not an automorphism, with associated growth data $(\rho, j)$. We choose a surface $W$ and morphisms $\phi: W \rightarrow X, \psi: W \rightarrow X$ such that $\sigma=\psi \phi^{-1}$; moreover we make this choice such that the rank of $N^{1}(W)$ is minimal. Choose any decomposition $\phi=$ $\phi_{1} \phi_{2} \ldots \phi_{m}$, where each $\phi_{i}$ is a single monoidal transformation, and let $E \subseteq W$ be the exceptional curve contracted by $\phi_{m}$. Then $V=\psi(E) \subseteq X$ is an irreducible curve which is contracted by $\sigma^{-1}$, and $(V . V) \geq-1$. Moreover, we have the following.

(1) If $(V . V)=-1$, then there exists a monoidal transformation $\pi: X \rightarrow X^{\prime}$ with exceptional curve $V$ (and in this case, if $\sigma$ is stable, then so is $\sigma^{\prime}=$ $\left.\pi \sigma \pi^{-1}: X^{\prime} \rightarrow X^{\prime}\right)$. 
(2) If $(V \cdot V)=0$, then there exists a rational fibration $f: X \rightarrow C$ such that $V \cong \mathbb{P}^{1}$ is a generic fiber.

Proof. The same proof as that of [DF, Proposition 1.7] works here; we have just organized the result differently by moving some parts of the proof into the statement. The proof of the existence of a rational fibration in case (2) depends on the classical result BPV, Proposition 4.3], which also is valid over an arbitrary algebraically closed field. Note that $\overline{\mathrm{DF}}$, Proposition 1.7] contains an extra hypothesis that $(C . C) \leq 0$ for all irreducible curves $C$ contracting under $\sigma^{-1}$ (such as $V$ ). This hypothesis is not needed in our version; we simply draw no conclusion if $(V . V)>0$.

Proposition 2.14 ([DF, Lemma 4.1]). Suppose that $\sigma: X \rightarrow X$ is a birational map, such that $\left\|\left(\sigma^{n}\right)^{*}\right\|$ is bounded. Then $\sigma$ is conjugate to an automorphism $\tau$ : $Y \rightarrow Y$ such that $\left(\tau^{n}\right)^{*}: N^{1}(Y) \rightarrow N^{1}(Y)$ is the identity for some $n \geq 1$.

Proof. The proof in $\overline{\mathrm{DF}}$, which is easy to rewrite in algebraic language, uses Theorem 2.9. Lemma 2.11, Lemma 2.3(2), and Lemma 2.13.

Proposition 2.15 ([DF, Lemma 4.2]). Let $\sigma: X \rightarrow X$ be birational, with growth data $(\rho, j)$ given by Definition 2.12 such that $\rho=1$. Then either $(1) \sigma$ is conjugate to an automorphism $\tau: Y \rightarrow Y$, or else (2) $j=1$ and $\sigma$ is conjugate to another birational map $\mu: Z \rightarrow Z$ such that (i) $\mu$ is stable; (ii) $Z$ has a rational fibration $f: Z \rightarrow C$ which is preserved by $\mu$; and (iii) if $S$ is the set of irreducible curves contracted by $\mu^{-1}$, then for all $V \in S$ we have $\mu^{*}(V)=V$ in $N^{1}(Z)$, and $\left(V_{i} \cdot V_{\ell}\right)=0$ for all $V_{i}, V_{\ell} \in S$.

Proof. This is a restatement and slight strengthening of the result of DF, Lemma 4.2 ], which does not require in case (2) the restrictions given in (iii). So we indicate the needed adjustments to the proof.

First, by Theorem 2.9 we replace $X$ with a blowup to assume $\sigma$ is stable. Suppose that $\sigma$ is not an automorphism. As in the statement of Lemma 2.13. choose a $W$ with Neron-Severi group of minimal rank which has morphisms $\phi: W \rightarrow X$, $\psi: W \rightarrow X$ such that $\sigma=\psi \phi^{-1}$. Given any point $p \in X$ which is a fundamental point for $\sigma$, we can choose a decomposition $\phi=\phi_{1} \phi_{2} \cdots \phi_{m}$ where each $\phi_{i}$ is a single monoidal transformation, and moreover such that the exceptional curve $E \subseteq W$ contracted by $\phi_{m}$ satisfies $\phi(E)=p$. Let $V=\psi(E)$. If $(V . V)=-1$, then using Lemma 2.13(1) we contract $V$ by a single monoidal transformation $\pi: X \rightarrow X^{\prime}$ such that $\sigma^{\prime}=\pi \sigma \pi^{-1}: X^{\prime} \rightarrow X^{\prime}$ is again stable. Replacing $X$ by $X^{\prime}$ and $\sigma$ by $\sigma^{\prime}$, we repeat this process as many times as possible. The process is finite since the rank of the Neron-Severi group decreases by one with each contraction.

If the process of the last paragraph results in an automorphism, we are done, so assume not. Then we have obtained a stable birational map $\sigma: X \rightarrow X$ such that the set $S$ of irreducible curves contracted by $\sigma^{-1}$ is nonempty. We can pick some fundamental point $p$ of $\sigma$ and repeat the first part of the previous paragraph; then $(V . V) \geq 0$ by Lemma 2.13, since we repeated the contraction process until no longer possible. Since $\rho=1$ and the proof of [DF, Lemma 4.2] (which depends on Lemma 2.10. Lemma 2.3(2), and Lemma 2.13) shows that $(V . V)=0$, there is a rational fibration $f: X \rightarrow C$ preserved by $\sigma$ with generic fiber $V, \sigma^{*}(V)=V$ in $N^{1}(X)$, and $j=1$. The union of the curves which are contracted by $\sigma^{-1}$ to the point $p$ is a connected set by Zariski's main theorem, and all of these curves lie in 
the same fiber of $f$ as $V$ since $\sigma$ preserves the fibration. But $V$ is a generic fiber, and so $V$ is the only curve contracted by $\sigma^{-1}$ to $p$. Moreover, any other $V^{\prime} \in S$ must also lie in some fiber and so $\left(V^{\prime} \cdot V\right)=0$.

Applying the previous paragraph to each fundamental point of $\sigma$, we see that each $V_{i} \in S$ contracts by $\sigma^{-1}$ to a unique point $p_{i}, \sigma^{*}\left(V_{i}\right)=V_{i}$ in $N^{1}(X)$, and $\left(V_{i} . V_{\ell}\right)=0$ for all $V_{\ell} \in S$.

\section{Geometricity}

In this section, a surface always means a nonsingular integral projective surface over the algebraically closed field $k$. In addition, $\sigma: X \rightarrow X$ continues to denote a birational map of a surface $X$.

Definition 3.1. If $K$ is a finitely generated field extension of $k$ with tr. $\operatorname{deg} K / k=2$ and if $\tau: K \rightarrow K$ is a field automorphism over $k$, we say that $\tau$ is geometric if there exists a surface $Z$ with $k(Z)=K$ such that the corresponding birational map $\tau: Z \rightarrow Z$ is an automorphism. Given a birational map $\sigma: X \rightarrow X$, we say that $\sigma$ is geometric if the corresponding field automorphism $\sigma: K \rightarrow K$ is geometric, or equivalently if $\sigma$ is conjugate (as defined in the previous section) to some automorphism $\tau: Z \rightarrow Z$ of a surface $Z$.

In [DF, $\sigma: X \rightarrow X$ is called bimeromorphically conjugate to an automorphism when we call it geometric. The following is a summary theorem of the main classification result in $[\mathrm{DF}]$.

Theorem 3.2 ([DF, Theorem 0.2]). Let $\sigma: X \rightarrow X$ be a birational map, and define the growth data $(\rho, j)$ by Definition 2.12 . Choose some matrix norm $\|\cdot\|$ on $\operatorname{End}_{\mathbb{C}}\left(N^{1}(X)_{\mathbb{C}}\right)$. Then exactly one of the following cases occurs:

(1) $\rho=1, j=0,\left\|\left(\sigma^{n}\right)^{*}\right\| \sim 1$, and $\sigma$ is geometric; in fact, $\sigma$ is conjugate to some automorphism $\tau: Y \rightarrow Y$ such that $\left(\tau^{n}\right)^{*}: N^{1}(Y) \rightarrow N^{1}(Y)$ is the identity for some $n \geq 1$.

(2) $\rho=1, j=1,\left\|\left(\sigma^{n}\right)^{*}\right\| \sim n, \sigma$ is not geometric, and $\sigma$ is conjugate to a birational map $\tau: Y \rightarrow Y$ where $Y$ has a rational fibration $f: Y \rightarrow C$ preserved by $\tau$.

(3) $\rho=1, j=2,\left\|\left(\sigma^{n}\right)^{*}\right\| \sim n^{2}$, and $\sigma$ is geometric.

(4) $\rho>1$, and $\left\|\left(\sigma^{n}\right)^{*}\right\| \sim n^{j} \rho^{n}$ is growing exponentially.

Proof. Most of this follows immediately from Proposition 2.14. Proposition 2.15] and Lemma 2.11. It only remains to show that if $\rho=1$ and $\sigma$ is geometric, then $j=0$ or 2 . But in this case we pass to a conjugate automorphism $\tau: Y \rightarrow Y$, and then the fact that $j=0$ or 2 is shown by Artin and Van den Bergh AV] Lemma 5.4]. For $k=\mathbb{C}$, this is also shown in [DF, Theorem 4.3, Proposition 4.4] (relying on previous work of Bellon, Cantat, and Gizatullin). In fact these results show that in case (3), it is even true that $\sigma$ preserves an elliptic fibration. We certainly expect that this also holds over an arbitrary algebraically closed field, though we have not checked since we will not need in the sequel that $\sigma$ preserves an elliptic fibration in case (3).

Theorem 3.2 completely determines whether a birational map $\sigma: X \rightarrow X$ with $\rho=1$ is geometric, in terms of the value of $j$; in particular, nongeometric such maps have a very restricted form. Proposition 3.5 below offers another characterization of geometricity, independent of the value of $\rho$. This gives a nice intuitive picture 
and will also be useful in our study of twisted section rings in the next section. In the following definition, we single out a pathology that turns out to characterize stable nongeometric maps.

Definition 3.3. Let $\sigma: X \rightarrow X$ be birational. Suppose that there exists a point $p \in X$ such that $\sigma^{-1}$ is defined at $p_{n}=\sigma^{-n}(p)$ for all $n \geq 0$, but $\sigma^{n}$ is not defined at $p$ for infinitely many $n>0$. Then we say that $p$ is an unbalanced point and that $\sigma$ is unbalanced. If no such $p$ exists, then we say that $\sigma$ is balanced.

Remark 3.4. The following easy observations, which are left to the reader, will be useful below. Let $\sigma: X \rightarrow X$ be birational. If $\sigma$ is unbalanced, then $\sigma^{n}: X--\rightarrow X$ is also unbalanced for every $n \geq 1$. Conversely, if $\sigma$ is stable and $\sigma^{n}$ is unbalanced for some $n \geq 1$, then $\sigma$ is unbalanced.

Proposition 3.5. Let $\sigma: X \rightarrow X$ be a birational map.

(1) If $\sigma$ is unbalanced, then $\sigma$ is not geometric.

(2) If $\sigma$ is stable, then $\sigma$ is balanced if and only if $\sigma$ is geometric.

Proof. (1) Let $\sigma: X \rightarrow X$ be unbalanced. As a first step, we will prove the following claim: suppose that $\phi: Z \rightarrow X$ is a birational morphism, and let $\tau=$ $\phi^{-1} \sigma \phi: Z \rightarrow Z$; then $\tau^{n}: Z \rightarrow Z$ is also unbalanced for some $n \geq 1$. Since $\phi$ is a composition of finitely many monoidal transformations, by induction it is enough to prove the claim in case $\phi$ is itself a single monoidal transformation, say with exceptional curve $E \subseteq Z$ and contracted point $q \in X$. Let $p \in X$ be an unbalanced point for $\sigma$, and define $p_{n}=\sigma^{-n}(p)$ for $n \geq 0$. If $p_{n} \neq q$ for all $n \geq 0$, then define $r=\phi^{-1}(p)$, while if $p_{n}=q$ for exactly one $n$, then define $r=\phi^{-1}\left(p_{n+1}\right)$. In either case it is now easy to check that $r$ is an unbalanced point for $\tau$, so $\tau$ itself is unbalanced. Otherwise, $p_{i}=p_{j}=q$ for some $0<i<j$. Let $d=j-i$; then $\sigma^{-d}$ is defined at $q$ with $\sigma^{-d}(q)=q$. Now suppose that $\sigma^{-d}$ is a local isomorphism at $q$. Then the inverse $\sigma^{d}$ is also defined at $q$, and since $\sigma^{n}(q)$ is defined for all $n \leq 0$, it follows that $\sigma^{n}(q)$ is defined for all $n \in \mathbb{Z}$. But then $\sigma^{n}(p)$ is defined for all $n \in \mathbb{Z}$, a contradiction. Thus $\sigma^{-d}$ is not a local isomorphism at $q$, so $\sigma^{-d}$ induces a nonsurjective map of tangent spaces $T_{q} \rightarrow T_{q}$. But because points of the exceptional curve $E$ of $\phi$ correspond to tangent directions at $q$, it follows that $\tau^{-d}(E)=r$ for some point $r \in E$. Moreover, since $\sigma^{-d}$ is defined at $q, \tau^{-d}$ is defined at every point of $E$ (for example, by the universal property of blowing up); in particular, at $r$. Thus $r$ is an unbalanced point for $\tau^{d}$, proving the claim.

Next, we claim that it is impossible to have an unbalanced birational map $\tau$ : $Z \rightarrow Z$ and a birational morphism $\psi: Z \rightarrow Y$ with $\mu=\psi \tau \psi^{-1}: Y \rightarrow Y$ an automorphism. Suppose this is possible, and choose such an example where $\psi$ is a composition of the minimal possible number $m$ of monoidal transformations (obviously $m>0$ ). Now let $q \in Z$ be an unbalanced point. Then $\tau^{n}$ fails to be defined at $q$ for infinitely many $n>0$, say for some sequence $0<n_{1}<n_{2}<\ldots$. Thus we can find irreducible curves $C_{1}, C_{2}, \cdots \subseteq Z$ such that $\tau^{-n_{i}}\left(C_{i}\right)=q$. Clearly we must have that $\psi\left(C_{i}\right)=r_{i}$ is a point for all $i$. Since $\psi$ contracts finitely many curves, we have $C_{i}=C_{j}$ for some $0<i<j$, and so $r_{i}=r_{j}=r$, say. Moreover, $\mu^{-n_{i}}(r)=\psi(q)=\mu^{-n_{2}}(r)$; letting $d=n_{2}-n_{1}$, we then have $\mu^{d}(r)=r$. Work now instead with $\tau^{\prime}=\tau^{d}: Z \rightarrow Z$ and $\mu^{\prime}=\mu^{d}: Y \rightarrow Y$. Then $\mu^{\prime}(r)=r$, so if $\pi: \tilde{Y} \rightarrow Y$ is the monoidal transformation blowing up $r$, then $\mu^{\prime}$ lifts to an automorphism $\nu=\pi^{-1} \mu^{\prime} \pi: \widetilde{Y} \rightarrow \widetilde{Y}$. Now $\psi=\pi \theta$ for some morphism $\theta: Z \rightarrow \tilde{Y}$, 
by [Ha, Proposition V.5.3]. But $\tau^{\prime}$ is again unbalanced (Remark 3.4) and descends to an automorphism $\nu=\theta \tau^{\prime} \theta^{-1}: \widetilde{Y} \rightarrow \widetilde{Y}$, where $\theta$ is composed of fewer than $m$ monoidal transformations. This contradicts the minimal choice of $m$ and this contradiction proves the claim.

Finally, suppose that $\sigma: X \rightarrow X$ is unbalanced and geometric. Then we may find a surface $Z$ and birational morphisms $\phi: Z \rightarrow X, \psi: Z \rightarrow Y$, such that $\mu=\psi \phi^{-1} \sigma \phi \psi^{-1}: Y \rightarrow Y$ is an automorphism. By the first claim, $\tau^{n}=\phi^{-1} \sigma^{n} \phi:$ $Z \rightarrow Z$ is unbalanced for some $n \geq 1$. Then by the second claim, $\mu^{n}$ is not an automorphism, a contradiction.

(2). Let $\sigma: X \rightarrow X$ be balanced and stable. Suppose that $\sigma$ is not already an automorphism (in which case we are done) and let $C \subseteq X$ be an irreducible curve with $\sigma(C)=p$ a point. By the stability condition, $\sigma^{n}(p)$ is defined for all $n \geq 0$, so at a point $r \in C$ for which $\sigma(r)$ is defined, we have that $\sigma^{n}(r)$ is defined for all $n \geq 0$. There are at most finitely many points of $C$ at which $\sigma$ is not defined; for one of these, say $q$, there must be a curve $D$ with $\sigma^{-1}(D)=q$. Then by the stability condition, $q_{n}=\sigma^{-n}(q)$ is defined for all $n \geq 0$, with $\sigma^{-1}$ defined at each $q_{n}$, implying that $\sigma^{n}(q)$ is defined for all $n \gg 0$ by the balanced hypothesis. In conclusion, $\sigma^{n}$ is defined at every point of $C$ for some $n \geq 1$. Then we can choose a nonsingular surface $Z$ and morphisms $\phi: Z \rightarrow X, \psi: Z \rightarrow X$ for which $\psi \phi^{-1}=\sigma^{n}$, and where $\phi^{-1}$ is a local isomorphism at every point of $C$ (see the proof of Ha, Theorem V.5.5]). Thus $(C . C)_{X}=\left(\phi^{-1}(C) \cdot \phi^{-1}(C)\right)_{Z} \leq-1$ since $\phi^{-1}(C)$ is a curve contracting under $\psi$. In conclusion, all irreducible curves of $X$ contracted by $\sigma$ have negative self-intersection. Now using Lemma 2.13 (applied to $\sigma^{-1}$ ) we can find a monoidal transformation $\pi: X \rightarrow X^{\prime}$ blowing down some irreducible curve contracted by $\sigma$, where replacing $X$ by $X^{\prime}$ and $\sigma$ by $\sigma^{\prime}=\pi \sigma \pi^{-1}, \sigma^{\prime}$ is still stable. Moreover, $\sigma^{\prime}$ is also balanced, by the first claim proved in the first half of the proof, together with Remark 3.4 .

Repeat the previous paragraph as many times as possible. It must reach an automorphism after finitely many steps since the rank of the Neron-Severi group decreases by 1 each time. Thus we have shown that for a stable map, balanced implies geometric, and the converse was proved in part (1).

The preceding proposition is useful for constructing examples of nongeometric maps.

Example 3.6. We thank Michael Artin for suggesting the following example. Assume that $k$ is uncountable. Let $X=\mathbb{P}^{2}$, let $\tau: \mathbb{P}^{2} \rightarrow \mathbb{P}^{2}$ be the Cremona map of Example 2.5 and let $\phi: \mathbb{P}^{2} \rightarrow \mathbb{P}^{2}$ be an automorphism. Let $\sigma=\tau \phi: \mathbb{P}^{2} \rightarrow \mathbb{P}^{2}$. Let $\ell_{1}, \ell_{2}, \ell_{3}$ be the coordinate lines which are contracted by $\tau$. Note that $\sigma^{-1}\left(\ell_{j}\right)=p_{j, 0}$ is a point for each $j$. Now if we choose $\phi$ generically (outside some countable union of proper closed subsets of $\operatorname{PGL}(2, k))$, we can ensure that $\sigma^{n}\left(\ell_{j}\right)$ is a curve for all $j$ and $n \geq 1$, and moreover that we can inductively define $p_{j, i+1}=\sigma^{-1}\left(p_{j, i}\right)$ for all $j$ and $i \geq 0$. Then $\sigma^{-1}$ and thus $\sigma$ is stable, and $\sigma$ is not geometric by Proposition 3.5 since each point $p_{j, 0}$ is unbalanced. Note also that $\operatorname{Pic} X=\mathbb{Z}$ and $\sigma^{*}:$ Pic $X \rightarrow \operatorname{Pic} X$ is multiplication by 2 . So $\rho=2>1$ for this stable birational map.

We remark that there also are geometric birational maps $\sigma: X \rightarrow X$ with $\rho>1$; an automorphism of a $K 3$ surface with $\rho>1$ is given in Ke1, Example 3.9]. Also, it is easy to find examples of case (2) of Theorem 3.2 in fact, any ruled 
surface has a nongeometric birational map preserving the ruling which is of this type (see DF, Remark 7.3]).

As a further application of Proposition 3.5. we have the following result that the property of geometricity is invariant under base extension.

Lemma 3.7. Let $k \subseteq L$ where $L$ is another algebraically closed field, let $K / k$ be a finitely generated field extension with $\operatorname{tr} . \operatorname{deg} K / k=2$, and let $\sigma \in \operatorname{Aut}_{k} K$. Let $F$ be the field of fractions of $K \otimes_{k} L$, so $\sigma$ extends uniquely to an automorphism $\widetilde{\sigma} \in \operatorname{Aut}_{L} F$. Then $\sigma \in \operatorname{Aut}_{k} K$ is geometric if and only if $\widetilde{\sigma} \in \mathrm{Aut}_{L} F$ is geometric.

Proof. It is a standard result that since $k$ is algebraically closed, $K \otimes_{k} L$ is a domain, so it makes sense to consider its field of fractions $F$. If $\sigma \in \mathrm{Aut}_{k} K$ is geometric, pick a projective model $X$ of $K$ such that the induced map $\sigma: X \rightarrow X$ is an automorphism. Then $\sigma$ lifts to an automorphism $\widetilde{\sigma}: \widetilde{X} \rightarrow \tilde{X}$ of the projective $L$ surface $\widetilde{X}=X \times_{\operatorname{Spec} k}$ Spec $L$, so the corresponding field automorphism $\widetilde{\sigma} \in \operatorname{Aut}_{L} F$ is again geometric.

Conversely, if $\sigma \in \mathrm{Aut}_{k} K$ is not geometric, we choose a nonsingular projective model $X$ of $K$ such that the corresponding birational map $\sigma: X \rightarrow X$ is stable, by Theorem 2.9. By Proposition 3.5(2), there is some unbalanced point $p \in X$ for $\sigma$; then it is easy to see that the lifted point $\widetilde{p} \in \widetilde{X}=X \times_{\operatorname{Spec} k} \operatorname{Spec} L$ is an unbalanced point for $\widetilde{\sigma}: \widetilde{X} \rightarrow \widetilde{X}$, so by Proposition $3.5(1), \widetilde{\sigma} \in \operatorname{Aut}_{L} F$ is not geometric either.

\section{TWisted SECTION RINGS}

If $\sigma: X \rightarrow X$ is an automorphism of a surface $X$, then given an invertible sheaf $\mathcal{L}$ on $X$ we may form the twisted homogeneous coordinate ring $B(X, \mathcal{L}, \sigma)$ as described in the introduction. Since $B_{n}=\mathrm{H}^{0}\left(X, \mathcal{L}_{n}\right)$ is the full vector space of global sections of an invertible sheaf on $X$, many questions about $B$ reduce to questions about invertible sheaves. For example, the calculation of the growth of $\operatorname{dim}_{k} B_{n}$ can make use of the Riemann-Roch theorem. In this section, we generalize this construction to form a ring $\widetilde{B}(X, \mathcal{L}, \sigma)$ where $\sigma: X \rightarrow X$ is just a stable birational map, and where $\widetilde{B}_{n}=\mathrm{H}^{0}\left(X, \mathcal{L}_{n}\right)$ is the vector space of global sections of the analogous invertible sheaf $\mathcal{L}_{n}$ on $X$. The growth of the graded pieces of $\widetilde{B}$ will still be a consequence of the Riemann-Roch theorem, and we give it in the next section. Unfortunately, for nongeometric $\sigma$ the $\operatorname{ring} \widetilde{B}$ is otherwise not wellbehaved: we will show in this case that (for $\mathcal{L}$ ample enough) $\widetilde{B}$ is not finitely generated as an algebra.

We now describe some notational conventions that will hold throughout this section. As in previous sections, $X$ will always be a nonsingular integral projective surface. Given an invertible sheaf $\mathcal{L}$ on $X$, for convenience we will assume that we have a fixed embedding $\mathcal{L} \subseteq \mathcal{K}$ of $\mathcal{L}$ in the constant sheaf $\mathcal{K}$ of rational functions $K=k(X)$; there is then a corresponding Weil divisor $D$ on $X$ with $\mathcal{L}=\mathcal{O}_{X}(D)$. Given some stable birational map $\sigma: X \rightarrow X$, we define the pullback map $\sigma^{*}$ : $\Pi c X \rightarrow \operatorname{Pic} X$ on Weil divisors as in Definition 2.1. Thinking in terms of invertible sheaves, we define $\sigma^{*} \mathcal{L}=\mathcal{O}_{X}\left(\sigma^{*}(D)\right)$, so there is also a fixed embedding $\sigma^{*} \mathcal{L} \subseteq \mathcal{K}$. As in the theory of twisted homogeneous coordinate rings, it is convenient to use the notation $\mathcal{L}^{\sigma}=\sigma^{*} \mathcal{L}$, and we define $\mathcal{L}_{0}=\mathcal{O}_{X}$ and $\mathcal{L}_{n}=\mathcal{L} \otimes \mathcal{L}^{\sigma} \otimes \cdots \otimes \mathcal{L}^{\sigma^{n-1}} \subseteq \mathcal{K}$ for $n \geq 1$. The birational map $\sigma: X \rightarrow X$ induces an automorphism $K \rightarrow K$, which we also call $\sigma$. Finally, we write $V^{\sigma}=\sigma(V)$ for any subset $V \subseteq K$. 
We begin by recording some simple sheaf-theoretic properties of the pullback map.

Lemma 4.1. Let $\sigma: X \rightarrow X$ be a stable birational map, and $\mathcal{L}$ an invertible sheaf on $X$. Choose a nonsingular surface $W$ with birational morphisms $\phi: W \rightarrow X$ and $\psi: W \rightarrow X$ such that $\sigma=\psi \phi^{-1}$.

(1) $\mathcal{L}^{\sigma} \subseteq \mathcal{K}$ is the reflexive hull $\mathcal{F}^{* *}=\mathcal{H o m}\left(\mathcal{H o m}\left(\mathcal{F}, \mathcal{O}_{X}\right), \mathcal{O}_{X}\right)$ of the sheaf $\mathcal{F}=\phi_{*} \psi^{*} \mathcal{L} \subseteq \mathcal{K}$.

(2) There is an induced injective pullback of sections map

$$
\sigma^{*}: \mathrm{H}^{0}(X, \mathcal{L}) \rightarrow \mathrm{H}^{0}\left(X, \mathcal{L}^{\sigma}\right)
$$

which is also the restriction of the automorphism $\sigma: K \rightarrow K$.

(3) If $V \subseteq \mathrm{H}^{0}(X, \mathcal{L})$ generates $\mathcal{L}$, then $V^{\sigma}$ generates $\mathcal{L}^{\sigma}$ except possibly at the finitely many fundamental points of $\sigma$. Similarly, $V V^{\sigma} \ldots V^{\sigma^{n-1}}$ generates $\mathcal{L}_{n}$ except at a finite set of points for all $n \geq 1$.

Proof. (1) The sheaf $\mathcal{F}=\phi_{*} \psi^{*} \mathcal{L}$ is locally free except possibly at the finite set $S$ of fundamental points of $\sigma$. The reflexive hull $\mathcal{F}^{* *}$ is the unique invertible sheaf on $X$ agreeing with $\mathcal{F}$ on $U=X \backslash S$ [RS1, Sublemma 7.7]. Now part (1) is just a reinterpretation of Definition 2.1 .

(2) Note that since we have embeddings $\mathcal{L} \subseteq \mathcal{K}$ and $\mathcal{L}^{\sigma} \subseteq \mathcal{K}$, taking sections we also have inclusions $\mathrm{H}^{0}(X, \mathcal{L}) \subseteq K$ and $\mathrm{H}^{0}\left(X, \mathcal{L}^{\sigma}\right) \subseteq K$. Now $\sigma^{*}$ : $\mathrm{H}^{0}(X, \mathcal{L}) \rightarrow \mathrm{H}^{0}\left(X, \mathcal{L}^{\sigma}\right)$ may be defined by composing the injective pullback map $\psi^{*}: \mathrm{H}^{0}(X, \mathcal{L}) \rightarrow \mathrm{H}^{0}\left(W, \psi^{*} \mathcal{L}\right)$, the bijective pushforward of sections map $\phi_{*}$ : $\mathrm{H}^{0}\left(W, \psi^{*} \mathcal{L}\right) \rightarrow \mathrm{H}^{0}(X, \mathcal{F})$, and the injection $\mathrm{H}^{0}(X, \mathcal{F}) \rightarrow \mathrm{H}^{0}\left(X, \mathcal{F}^{* *}\right)$ induced by the canonical injection of sheaves $\mathcal{F} \subseteq \mathcal{F}^{* *} \subseteq \mathcal{K}$. It follows formally that $\sigma^{*}$ is also given by restricting $\sigma: K \rightarrow K$.

(3) By the construction of $\sigma^{*}$ in part (2), it is clear that $\sigma^{*}(V)=V^{\sigma} \subseteq \mathrm{H}^{0}(X, \mathcal{F})$ generates the sheaf $\mathcal{F}$ except possibly at the finite set $S$ of fundamental points of $\sigma$. The injection $\mathcal{F} \subseteq \mathcal{F}^{* *}=\mathcal{L}^{\sigma}$ is an isomorphism at points not in $S$, so $V^{\sigma}$ generates $\mathcal{L}^{\sigma}$ at points not in $S$. Applying this to each power $\sigma^{i}$, we see that $V^{\sigma^{i}}$ generates $\mathcal{L}^{\sigma^{i}}$ at points not in the set $S_{i}$ of fundamental points of $\sigma^{i}$; thus $V V^{\sigma} \ldots V^{\sigma^{n-1}}$ generates $\mathcal{L}_{n}$ except possibly at points of the finite set $\bigcup_{i=1}^{n-1} S_{i}$.

Definition 4.2. Given a stable birational map $\sigma: X \rightarrow X$ and $\mathcal{L} \subseteq \mathcal{K}$ an invertible sheaf, we define the twisted section ring

$$
\widetilde{B}(X, \mathcal{L}, \sigma)=\bigoplus_{n \geq 0} \mathrm{H}^{0}\left(X, \mathcal{L}_{n}\right) t^{n} \subseteq Q=K\left[t, t^{-1} ; \sigma\right],
$$

with multiplication induced by that of $Q$.

Note that if $\sigma$ is an automorphism, then $\widetilde{B}(X, \mathcal{L}, \sigma) \cong B(X, \mathcal{L}, \sigma)$ is the usual twisted homogeneous coordinate ring. Defining $\widetilde{B}$ as an explicit subring of $Q$ is not really necessary, but it avoids a tedious proof that the multiplication of $\widetilde{B}$ is associative. Still, to ensure that Definition 4.2 actually defines a ring, we need to check that $\widetilde{B}$ is closed under multiplication. For this, it is enough to check that $\widetilde{B}_{m} \widetilde{B}_{n} \subseteq \widetilde{B}_{m+n}$ for $m, n \geq 0$. If $f \in \mathrm{H}^{0}\left(X, \mathcal{L}_{m}\right)$ and $g \in \mathrm{H}^{0}\left(X, \mathcal{L}_{n}\right)$, then $f t^{m} g t^{n}=$ $f \sigma^{m}(g) t^{m+n}$ and $\sigma^{m}(g)=\left(\sigma^{m}\right)^{*}(g) \in \mathrm{H}^{0}\left(X, \mathcal{L}_{n}^{\sigma^{m}}\right)$ by part (2) of Lemma 4.1. Now 
since $\sigma$ is stable, we have

$$
\begin{aligned}
\mathcal{L}_{n}^{\sigma^{m}} & =\left(\sigma^{m}\right)^{*}\left(\mathcal{L} \otimes \sigma^{*} \mathcal{L} \otimes \cdots \otimes\left(\sigma^{n-1}\right)^{*} \mathcal{L}\right) \\
& =\left(\sigma^{m}\right)^{*} \mathcal{L} \otimes\left(\sigma^{m}\right)^{*} \sigma^{*}(\mathcal{L}) \otimes \cdots \otimes\left(\sigma^{m}\right)^{*}\left(\sigma^{n-1}\right)^{*} \mathcal{L} \\
& =\left(\sigma^{m}\right)^{*} \mathcal{L} \otimes\left(\sigma^{m+1}\right)^{*}(\mathcal{L}) \otimes \cdots \otimes\left(\sigma^{n+m-1}\right)^{*} \mathcal{L},
\end{aligned}
$$

and so $\mathcal{L}_{m} \otimes \mathcal{L}_{n}^{\sigma^{m}}=\mathcal{L}_{m+n}$, as subsheaves of $\mathcal{K}$. Then $f \sigma^{m}(g)$ is the image of the element $f \otimes\left(\sigma^{m}\right)^{*}(g)$ under the multiplication map

$$
\mathrm{H}^{0}\left(X, \mathcal{L}_{m}\right) \otimes \mathrm{H}^{0}\left(X, \mathcal{L}_{n}^{\sigma^{m}}\right) \rightarrow \mathrm{H}^{0}\left(X, \mathcal{L}_{m+n}\right),
$$

so $\widetilde{B}$ is indeed a subalgebra of $Q$.

The goal of the rest of the section is to prove that the $\operatorname{ring} \widetilde{B}(X, \mathcal{L}, \sigma)$ is typically not finitely generated as a $k$-algebra if $\sigma$ is nongeometric. The exact theorem will hold only for $\mathcal{L}$ "ample enough". We next define an appropriate such class of invertible sheaves. The definition is fairly arbitrary; it is made just for the convenience of this paper to include enough positivity properties for several later results.

Definition 4.3. Given a stable birational map $\sigma: X \rightarrow X$, we say an invertible sheaf $\mathcal{L}$ on $X$ is $\sigma$-positive if (1) $\mathcal{L}$ is ample, (2) the sheaf $\mathcal{L}_{n}$ is generated by its global sections for all $n \geq 1$, and $(3) \mathrm{H}^{i}\left(X, \mathcal{L}_{n}\right)=0$ for all $i>0$ and $n \geq 1$.

We note that it is easy to find $\sigma$-positive invertible sheaves.

Lemma 4.4. Given $\sigma: X \rightarrow X$ stable and a very ample invertible sheaf $\mathcal{M}$ on $X$, then $\mathcal{L}=\mathcal{M}^{\otimes d}$ is $\sigma$-positive for all $d \gg 0$.

Proof. The proof uses the notion of Castelnuovo-Mumford regularity with respect to the very ample sheaf $\mathcal{M}$, and is mostly a matter of quoting some known results. We recall the definition: for a coherent sheaf $\mathcal{F}$ on $X, \mathcal{F}$ is called $d$-regular if $\mathrm{H}^{i}\left(X, \mathcal{F} \otimes \mathcal{M}^{\otimes d-i}\right)=0$ for all $i>0$. It is a fact that if $\mathcal{F}$ is $d$-regular, then $\mathcal{F}$ is $e$-regular for all $e \geq d[\mathrm{La}$, Theorem 1.8.5]. We let reg $\mathcal{F}$ be the smallest $d$ for which $\mathcal{F}$ is $d$-regular.

The formula $\lim _{n \rightarrow \infty} \operatorname{reg} \mathcal{M}^{\otimes n}=-\infty$ easily follows since $\mathcal{M}$ is ample. By a theorem of Fujita, there is a fixed bound $M$ such that reg $\mathcal{F} \leq M$ for all nef invertible sheaves $\mathcal{F}[\mathrm{Fj}$, Theorem 1, p. 520]. Also, there is a constant $C$ such that for any two invertible sheaves $\mathcal{N}, \mathcal{P}$ on $X$, one has $\operatorname{reg}(\mathcal{N} \otimes \mathcal{P}) \leq \operatorname{reg} \mathcal{N}+\operatorname{reg} \mathcal{P}+C$ Ke2, Proposition 2.8].

For $d \geq 1$ put $\mathcal{L}=\mathcal{M}^{\otimes d}$. Then $\mathcal{L}$ is nef and so $\left(\mathcal{L}^{\sigma} \otimes \cdots \otimes \mathcal{L}^{\sigma^{n-1}}\right)$ is nef by Lemma 2.2. Then for $d \gg 0$ we have $\operatorname{reg} \mathcal{L} \leq-C-M$ and so

$$
\operatorname{reg} \mathcal{L}_{n} \leq \operatorname{reg} \mathcal{L}+\operatorname{reg}\left(\mathcal{L}^{\sigma} \otimes \cdots \otimes \mathcal{L}^{\sigma^{n-1}}\right)+C \leq 0 .
$$

Thus $\mathrm{H}^{i}\left(X, \mathcal{L}_{n}\right)=0$ for $i=1,2$. Also, since $\mathcal{L}_{n}$ is 0 -regular, it is generated by its global sections [La, Theorem 1.8.5 and Remark 1.8.14].

Theorem 4.5. Let $\sigma: X \rightarrow X$ be stable and nongeometric, and suppose that $\mathcal{L} \subseteq \mathcal{K}$ is $\sigma$-positive. Then $\widetilde{B}(X, \mathcal{L}, \sigma)$ is not a finitely generated $k$-algebra.

Proof. Since $\sigma$ is not geometric, by Proposition 3.5 there must exist an unbalanced point $q \in X$, so $q$ is a fundamental point of $\sigma^{i}$ for infinitely many $i \geq 1$. We show first that in fact we can find a point which is a fundamental point for all positive powers of $\sigma$. Suppose we have $0<i_{1}<i_{2}<i_{3}$ such that $\sigma^{i_{1}}$ and $\sigma^{i_{3}}$ are not 
defined at $q$, but $\sigma^{i_{2}}(q)$ is defined. Then we can find irreducible curves $F_{1}$ and $F_{3}$ such that $\sigma^{-i_{1}}\left(F_{1}\right)=q$ and $\sigma^{-i_{3}}\left(F_{3}\right)=q$, so $\sigma^{i_{2}-i_{1}}\left(F_{1}\right)=\sigma^{i_{2}}(q)=\sigma^{i_{2}-i_{3}}\left(F_{3}\right)$, and this contradicts the fact that $\sigma$ is stable. Thus $\sigma^{i}$ is undefined at $q$ for all $i \gg 0$. Let $i_{0}$ be the largest nonnegative integer such that $p=\sigma^{i_{0}}(q)$ is defined. Then $p$ is a fundamental point of $\sigma^{i}$ for all $i \geq 1$. For each $i \geq 1$ we can pick an irreducible curve $E_{i} \subseteq X$ such that $\sigma^{-i}\left(E_{i}\right)=p$.

Now fix $n \geq 2$ and consider the multiplication map $\widetilde{B}_{i} \otimes \widetilde{B}_{n-i} \rightarrow \widetilde{B}_{n}$ for some $0<i<n$. Dropping the powers of $t$, this may be identified with

$$
\theta_{i}: \mathrm{H}^{0}\left(X, \mathcal{L}_{i}\right) \otimes \mathrm{H}^{0}\left(X, \mathcal{L}_{n-i}\right) \stackrel{1 \otimes\left(\sigma^{i}\right)^{*}}{\longrightarrow} \mathrm{H}^{0}\left(X, \mathcal{L}_{i}\right) \otimes \mathrm{H}^{0}\left(X, \mathcal{L}_{n-i}^{\sigma^{i}}\right) \longrightarrow \mathrm{H}^{0}\left(X, \mathcal{L}_{n}\right),
$$

where the second map is the natural multiplication map. We claim that the image of $\theta_{i}$ is contained in $\mathrm{H}^{0}\left(X, \mathcal{I}_{p} \otimes \mathcal{L}_{n}\right)$, where $\mathcal{I}_{p}$ is the ideal sheaf of the point $p$. Suppose for the moment that the claim has been proved for all $i$. Then we will have that the image of $\bigoplus_{i=1}^{n-1} \widetilde{B}_{i} \otimes \widetilde{B}_{n-i} \rightarrow \widetilde{B}_{n}$ under the multiplication map is contained in $\mathrm{H}^{0}\left(X, \mathcal{I}_{p} \otimes \mathcal{L}_{n}\right) t^{n}$. Since $\mathcal{L}$ is $\sigma$-positive, $\mathcal{L}_{n}$ is generated by global sections at $p$ and so $\mathrm{H}^{0}\left(X, \mathcal{I}_{p} \otimes \mathcal{L}_{n}\right) \subsetneq \mathrm{H}^{0}\left(X, \mathcal{L}_{n}\right)$. It follows that $\widetilde{B}$ is not generated in degrees 1 through $n-1$, and since $n \geq 2$ was arbitrary the result follows.

It remains to prove the claim. For this, it will be enough to prove that the image of the pullback of sections map $\left(\sigma^{i}\right)^{*}: \mathrm{H}^{0}\left(X, \mathcal{L}_{n-i}\right) \rightarrow \mathrm{H}^{0}\left(X, \mathcal{L}_{n-i}^{\sigma^{i}}\right)$ is contained in $\mathrm{H}^{0}\left(X, \mathcal{I}_{p} \otimes \mathcal{L}_{n-i}^{\sigma^{i}}\right)$. Let $\mathcal{N}=\left(\mathcal{L}^{\sigma} \otimes \cdots \otimes \mathcal{L}^{\sigma^{n-i-1}}\right)$ and note that $\mathcal{N}$ is nef by Lemma 2.2. Then $\mathcal{L}_{n-i}=\mathcal{L} \otimes \mathcal{N}$ is the product of an ample and a nef sheaf, so is also ample. Write $\mathcal{L}_{n-i}=\mathcal{O}_{X}(D)$ for some Weil divisor $D$. Given a section $0 \neq s \in \mathrm{H}^{0}\left(X, \mathcal{L}_{n-i}\right)$, let $D^{\prime} \in|D|$ be the divisor of zeroes of $s$, where $|D|$ is the complete linear system of effective divisors linearly equivalent to $D$. Note that since $\mathcal{L}_{n-i}$ is generated by its sections and obviously $\mathcal{L}_{n-i} \neq \mathcal{O}_{X}$, we have $\operatorname{dim}_{k} \mathrm{H}^{0}\left(X, \mathcal{L}_{n-i}\right) \geq 2$. It follows that for a generic $D^{\prime} \in|D|, D^{\prime}=\sum a_{j} C_{j}$ for some irreducible curves $C_{j}$, where (i) no $C_{j}$ contracts under $\sigma^{-i}$, and (ii) no $C_{j}$ contains a fundamental point of $\sigma^{-i}$. We will prove that for all such generic $D^{\prime}$, the support of the divisor $\left(\sigma^{i}\right)^{*}\left(D^{\prime}\right)$ contains $p$; then $\left(\sigma^{i}\right)^{*}(s) \in \mathrm{H}^{0}\left(X, \mathcal{I}_{p} \otimes \mathcal{L}_{n-i}^{\sigma^{i}}\right)$ will hold for generic $s \in \mathrm{H}^{0}\left(X, \mathcal{L}_{n-i}\right)$, so for all $s$ as required. Thus, let $D^{\prime} \in|D|$ with $D^{\prime}=\sum a_{j} C_{j}$ satisfying conditions (i) and (ii). Since $D^{\prime}$ is ample we have $\left(D^{\prime} . E_{i}\right)>0$. Furthermore, $C_{j} \neq E_{i}$ for each $j$, so some $C_{j}$ intersects $E_{i}$ nontrivially; but then $\sigma^{-i}$ is defined at every point of $C_{j}$, and $\sigma^{-i}\left(C_{j}\right)$ is a curve in the support of $\left(\sigma^{i}\right)^{*}\left(D^{\prime}\right)$ which contains the point $\sigma^{-i}\left(E_{i}\right)=p$.

The preceding theorem is not needed in the proof of the main theorem of the paper, but we have included it as an interesting negative result. As part of the program to classify noncommutative projective surfaces, one would like to describe in terms of geometry all connected finitely generated $\mathbb{N}$-graded subalgebras $A$ of $Q=K\left[t, t^{-1} ; \sigma\right]$, where $K / k$ is a finitely generated field extension of transcendence degree 2. For field automorphisms $\sigma$ which are geometric, the paper [RS1] succeeds in this goal, for those $A$ which are also Noetherian and generated in degree 1: such an $A$ is equal (in large degree) either to a twisted homogeneous coordinate ring $B(X, \mathcal{L}, \sigma)$, where $X$ is a surface, or else to a special kind of subring $R\left(X, Z_{\mathcal{I}}, \mathcal{L}, \sigma\right)=\bigoplus_{n \geq 0} \mathrm{H}^{0}\left(X, \mathcal{I}_{n} \otimes \mathcal{L}_{n}\right)$ of such a $B$ called a naïve blowup as studied in [KRS] and [RS2] (where here $\mathcal{I}_{n}=\mathcal{I} \cdot \sigma^{*} \mathcal{I} \cdots\left(\sigma^{n-1}\right)^{*} \mathcal{I}$ for some ideal sheaf $\mathcal{I}$ defining a 0 -dimensional subscheme $Z_{\mathcal{I}}$ of $X$ ). When $\sigma$ is not geometric, we do not know how to describe the subrings $A$ of $Q$. Theorem 4.5 shows that in this case the 
natural analogs of twisted homogeneous coordinate rings, the algebras $\widetilde{B}(X, \mathcal{L}, \sigma)$, unfortunately do not suffice to describe any such $A$. A different approach seems to be needed to understand the nongeometric case; we hope to address this question in future work.

\section{Growth OF $\widetilde{B}$}

In this section, we calculate the growth of the graded pieces of a $\operatorname{ring} \widetilde{B}(X, \mathcal{L}, \sigma)$ using the Riemann-Roch formula. For the case where $\sigma$ is nongeometric, we will also need to rely on the information from the classification result Theorem 3.2. The case where $\sigma$ is an automorphism is already known (see [AV] Theorem 1.7] or [Ke1, Theorem 6.1]), but we give a uniform proof that works in all cases since this takes little extra effort.

The following is the situation we always consider from now on.

Notation 5.1. Let $\sigma \in \operatorname{Aut}_{k}(K)$, where $K / k$ is a finitely generated field extension of an algebraically closed field $k$, with $\operatorname{tr} \cdot \operatorname{deg} K / k=2$. Choose a nonsingular integral projective surface $X$ over $k$ with $k(X)=K$ such that the induced map $\sigma: X \rightarrow X$ is stable (which is possible by Theorem 2.9). Fix some basis of $N^{1}(X) \cong \mathbb{Z}^{d}$ and let $P \in M_{d}(\mathbb{Z})$ be the $d \times d$ matrix representing the pullback map $\sigma^{*}: N^{1}(X) \rightarrow N^{1}(X)$. Fix any matrix norm $\|\cdot\|$ on $M_{d}(\mathbb{C})$, so $\left\|P^{n}\right\| \sim n^{j} \rho^{n}$ where $(\rho, j)$ is the associated growth data (which is independent of the choice of $X$ by Lemma 2.11). Let $\mathcal{K}$ be the constant sheaf of rational functions on $X$.

In some of our growth calculations, it will be convenient to assume the following further restrictions on the choice of $X$ :

Hypothesis 5.2. Assume Notation 5.1. If $\sigma$ is geometric, then by definition we can choose $X$ so that the map $\sigma: X \rightarrow X$ is an automorphism. If $\sigma$ is nongeometric with $\rho=1$, then by Proposition 2.15) (2) we have $j=1$ and we can choose $X$ so that $\sigma: X \rightarrow X$ is stable; $\sigma$ preserves some rational fibration $f: X \rightarrow C$; and if $S$ is the set of irreducible curves contracted by $\sigma^{-1}$, then $P\left(V_{i}\right)=V_{i}$ in $N^{1}(X)$ and $\left(V_{i} \cdot V_{\ell}\right)=0$ for all $V_{i}, V_{\ell} \in S$.

We need the following simple linear algebra lemma. The proof is similar to the proof of Lemma 2.11 and is left to the reader.

Lemma 5.3. Fix Notation 5.1, and let $Q \in M_{d}(\mathbb{R})$ be an invertible matrix.

(1) $\left\|Q P^{n}\right\| \sim n^{j} \rho^{n}$.

(2) For any two column vectors $v, w \in \mathbb{R}^{d},\left|v^{T} Q P^{n} w\right| \preccurlyeq n^{j} \rho^{n}$.

(3) There is a dense subset $U \subseteq \mathbb{R}^{d}$ (in fact, one can take $U$ to be the complement of some quadric hypersurface) such that for any $v \in U$, one has $\left|v^{T} Q P^{n} v\right| \sim n^{j} \rho^{n}$.

We now prove a series of growth estimates for the iterates of divisor classes under the pullback map $P$.

Lemma 5.4. Assume Notation 5.1 .

(1) For any $D, E \in N^{1}(X)_{\mathbb{R}}$, we have $\left|\left(P^{n} D . E\right)\right| \preccurlyeq n^{j} \rho^{n}$.

(2) For any ample $E \in N^{1}(X)_{\mathbb{R}},\left(P^{n} E . E\right) \sim n^{j} \rho^{n}$.

Proof. (1) For a review of the basic facts concerning intersection theory on a surface, see [Ha, Section V.1]. In particular, it is standard that the intersection form on $X$ is 
given by some symmetric real invertible matrix $Q$, such that for any column vectors $D, E \in N^{1}(X)_{\mathbb{R}} \cong \mathbb{R}^{d}$ we have $(D . E)=D^{T} Q E$. Then $\left(P^{n} D \cdot E\right)=E^{T} Q P^{n} D$ and the result follows from Lemma $5.3(2)$.

(2) As in part (1), let $Q$ be the matrix of the intersection form. Let $E$ be any ample divisor, and let $U$ be the open subset of Lemma 5.3(3). Since the nef cone $\operatorname{Nef}(X) \subseteq N^{1}(X)_{\mathbb{R}}$ is a cone of full dimension $d$ in $\mathbb{R}^{d}$, we can choose some $C \in U$ which is nef. We can also choose $m \gg 0$ so that $D=m E-C$ is nef. We have

$$
\left(P^{n} C . C\right)=\left(P^{n}(m E-D) \cdot m E-D\right)=m^{2}\left(P^{n} E . E\right)-\left(P^{n} D \cdot C\right)-m\left(P^{n} E . D\right) .
$$

Since $D$ and $C$ are nef by construction, and $P^{n} D, P^{n} E$, and $P^{n} C$ are nef by Lemma 2.2. (5.1) implies that $m^{2}\left(P^{n} E . E\right) \geq\left(P^{n} C . C\right) \geq 0$ for all $n$. Using that $C \in U$, we have $\left(P^{n} C . C\right) \sim n^{j} \rho^{n}$ by Lemma 5.3(3). Together with part (1) it follows that $\left(P^{n} E . E\right) \sim n^{j} \rho^{n}$ as desired.

Lemma 5.5. Assume that $\rho=1$ and that Hypothesis 5.2 holds. Let $D$ be an ample divisor on $X$ and set $D_{n}=\sum_{i=0}^{n-1} P^{i}(D)$. Then $\left(D_{n} . D_{n}\right) \sim n^{j+2}$.

Proof. We claim first that for any $D \in N^{1}(X)$ there is an integer constant $N \geq 0$ such that $\left(P^{b}(D) \cdot P^{a}(D)\right)=\left(P^{b-a}(D) \cdot D\right)+a N$ for any $0 \leq a \leq b$. If $\sigma$ is geometric, then we have chosen $\sigma: X \rightarrow X$ to be an automorphism in Hypothesis 5.2 . In this case, $P$ is an invertible matrix preserving the intersection form and so the claim obviously follows, with $N=0$.

Now suppose that $\sigma$ is nongeometric. Then $j=1$ and recall that we have assumed that $X$ is chosen in Hypothesis 5.2 so that if $S$ is the set of irreducible curves contracted by $\sigma^{-1}$, then $P\left(V_{i}\right)=V_{i}$ in $N^{1}(X)$ and $\left(V_{i} \cdot V_{\ell}\right)=0$ for all $V_{i}, V_{\ell} \in S$. Define the classes $E_{i}$ for the map $\sigma: X \rightarrow X$ as in Lemma 2.3, each $E_{i}$ is a sum with nonnegative coefficients of irreducible curves in $S$, and so $P\left(E_{i}\right)=E_{i}$ in $N^{1}(X)$ and $\left(E_{i} \cdot E_{\ell}\right)=0$ for all $i, \ell$. Then by Lemma 2.3(1), we see that for any class $B \in N^{1}(X)$, we have

$$
\left(P B \cdot E_{i}\right)=\left(P B \cdot P E_{i}\right)=\left(B \cdot E_{i}\right)+\sum_{\ell=1}^{d}\left(B \cdot E_{\ell}\right)\left(E_{i} \cdot E_{\ell}\right)=\left(B \cdot E_{i}\right) .
$$

Now for any $0 \leq a \leq b$, by Lemma 2.3 (1), we have

$$
\left(P^{b} D \cdot P^{a} D\right)=\left(P^{b-1} D \cdot P^{a-1} D\right)+\sum_{i=1}^{d}\left(P^{b-1} D \cdot E_{i}\right)\left(P^{a-1} D \cdot E_{i}\right),
$$

and by the previous calculation we also have

$$
\sum_{i=1}^{d}\left(P^{b-1} D \cdot E_{i}\right)\left(P^{a-1} D \cdot E_{i}\right)=\sum_{i=1}^{d}\left(D \cdot E_{i}\right)^{2} .
$$

Taking $N=\sum_{i=1}^{d}\left(D \cdot E_{i}\right)^{2}$, the claim follows by induction on $a$, the base case $a=0$ being trivial. 
Now let $D \in N^{1}(X)$ be ample. Using the claim of the first part of the proof we calculate

$$
\begin{aligned}
& \left(D_{n} \cdot D_{n}\right)=\sum_{i=0}^{n-1} \sum_{\ell=0}^{n-1}\left(P^{i} D \cdot P^{\ell} D\right)=\sum_{i=0}^{n-1}\left(P^{i} D \cdot P^{i} D\right)+\sum_{0 \leq i<\ell \leq n-1} 2\left(P^{i} D \cdot P^{\ell} D\right) \\
& =n(D . D)+\sum_{i=0}^{n-1} i N+\sum_{m=1}^{n-1} 2(n-m)\left(D \cdot P^{m} D\right)+\sum_{m=1}^{n-1}(n-m-1)(n-m) N .
\end{aligned}
$$

Obviously $n(D . D) \sim n$ and $\sum_{i=0}^{n-1} i N \sim N n^{2}$. Using the result of Lemma 5.4. we get the growth estimate

$$
\sum_{m=1}^{n-1} 2(n-m)\left(D \cdot P^{m} D\right) \sim \sum_{m=1}^{n-1} 2(n-m) m^{j} \sim n^{j+2} .
$$

Finally, $\sum_{m=1}^{n-1}(n-m-1)(n-m) N \sim N n^{3}$. To conclude, if $\sigma$ is geometric (so $N=0)$, then $\left(D_{n} . D_{n}\right) \sim n^{j+2}$. If $\sigma$ is nongeometric, then $j=1$ and $N \neq 0$ and we have $\left(D_{n} . D_{n}\right) \sim n^{3}=n^{j+2}$ in this case as well.

We now calculate the growth of the pieces of $\widetilde{B}(X, \mathcal{L}, \sigma)$ (for sufficiently positive $\mathcal{L})$. We will see later that this growth is exponential if $\rho>1$; here we just consider the case $\rho=1$.

Proposition 5.6. Assume Hypothesis 5.2 and that $\rho=1$. Let $\mathcal{L} \subseteq \mathcal{K}$ be a $\sigma$ positive invertible sheaf on $X$, and let $\widetilde{B}=\widetilde{B}(X, \mathcal{L}, \sigma)$. Then $\operatorname{dim}_{k} \widetilde{B}_{n} \sim n^{j+2}$.

Proof. We have $\widetilde{B}_{n} \cong \mathrm{H}^{0}\left(X, \mathcal{L}_{n}\right)$ by definition and $\mathrm{H}^{i}\left(X, \mathcal{L}_{n}\right)=0$ for $n \geq 1$ and $i>0$ by the $\sigma$-positive hypothesis. Writing $\mathcal{L}=\mathcal{O}_{X}(D)$, we have $\mathcal{L}_{n}=\mathcal{O}_{X}\left(D_{n}\right)$, where $D_{n}=\sum_{i=0}^{n-1} P^{i}(D)$. By the Riemann-Roch formula, for $n \geq 1$ we have

$$
\operatorname{dim}_{k} \mathrm{H}^{0}\left(X, \mathcal{L}_{n}\right)=\left(D_{n} . D_{n}\right) / 2-\left(D_{n} . K\right) / 2+1+p_{a},
$$

where $K$ is the canonical divisor on $X$ and $p_{a}$ is the arithmetic genus. By Lemma 5.5 . we have $\left(D_{n} . D_{n}\right) \sim n^{j+2}$ since $D$ is ample. Moreover, by Lemma 5.4 we have $\left|\left(P^{n}(D) . K\right)\right| \preccurlyeq n^{j}$ and thus $\left|\left(D_{n} . K\right)\right| \preccurlyeq n^{j+1}$. So $\operatorname{dim}_{k} \mathrm{H}^{0}\left(X, \mathcal{L}_{n}\right) \sim n^{j+2}$ as required.

\section{THE LOWER BOUND}

We begin this section by recalling some definitions concerning graded rings and their growth; see [KL] for more details. Given any finitely generated $k$-algebra $R$, if $V$ is a finite-dimensional generating subspace for $R$ containing 1, then the GelfandKirillov dimension of $R$ is GK $R=\varlimsup \log _{n} \operatorname{dim}_{k} V^{n}$ (which does not depend on $V)$. The algebra $R$ is said to have exponential growth if $\overline{\lim }\left(\operatorname{dim}_{k} V^{n}\right)^{1 / n}>1$. If $R$ is not finitely generated as an algebra, then GK $R$ is defined to be the supremum of GK $R^{\prime}$ for finitely generated subalgebras $R^{\prime} \subseteq R$.

Throughout $\mathbb{N}=\{0,1,2, \ldots\}$ denotes the nonnegative integers. Let $A$ be an $\mathbb{N}$-graded $k$-algebra; $A$ is called locally finite if $\operatorname{dim}_{k} A_{n}<\infty$ for all $n \geq 0$. If $A$ is a locally finite $\mathbb{N}$-graded $k$-algebra which is also finitely generated as a $k$-algebra, then GK $A=\left(\overline{\lim } \log _{n} \operatorname{dim}_{k} A_{n}\right)+1$ (see [KL, Lemma 6.1]) and moreover $A$ has exponential growth if and only if $\overline{\lim }\left(\operatorname{dim}_{k} A_{n}\right)^{1 / n}>1$. 
The goal we are heading towards is to understand the growth of finitely generated $\mathbb{N}$-graded subalgebras $A \subseteq Q=K\left[t, t^{-1} ; \sigma\right]$, where $K / k$ is a finitely generated field extension of transcendence degree 2. An arbitrary such $A \subseteq Q$ could be quite small (for example, if generated as an algebra by a single element, isomorphic to a polynomial ring in one variable) and so we concentrate on those $A$ which are large in $Q$ in the sense of the following definition from [RZ.

Definition 6.1. A locally finite $\mathbb{N}$-graded subalgebra $A=\bigoplus_{n=0}^{\infty} V_{n} t^{n} \subseteq Q=$ $K\left[t, t^{-1} ; \sigma\right]$ is called a big subalgebra of $Q$ if there is some $n \geq 1$ and an element $u \in V_{n}$ such that $K$ is the field of fractions of its subalgebra $k\left[V_{n} u^{-1}\right]$.

To find a lower bound for the GK-dimension of a big finitely generated $\mathbb{N}$-graded algebra $A \subseteq Q=K\left[t, t^{-1} ; \sigma\right]$, the idea is to compare $A$ with rings of the form $\widetilde{B}(X, \mathcal{L}, \sigma)$, the growth of which we calculated in the last section. If $\sigma$ is geometric then this is easy and is already accomplished in [RZ, Proposition 5.5(2)]: in this case any big $A$ contains an isomorphic copy of some twisted homogeneous coordinate ring $B(X, \mathcal{L}, \sigma)$, and the lower bound is immediate. When $\sigma$ is nongeometric, however, there is no obvious reason that $A$ should contain a copy of some $\widetilde{B}(X, \mathcal{L}, \sigma)$; in fact this seems highly unlikely since $\widetilde{B}$ is typically infinitely generated. In any case, the same proof as in [RZ, Proposition 5.5(2)] does not work and so we use a different tactic to show that $A$ is not growing more slowly than $\widetilde{B}$ : we restrict all divisors to a generic curve $E$ on $X$ and calculate a lower bound for the growth there using some combinatorial estimates.

First, we need a simple analytic lemma about the growth of functions satisfying a certain recurrence relation. Recall the equivalence relation on growth functions introduced before Lemma 2.10

Lemma 6.2. Let $f(n): \mathbb{N} \rightarrow \mathbb{R}_{>0}$ be a sequence of positive real numbers which satisfies the relation $f(n+1) \geq f(n)+f(m(n))$ for some function $m: \mathbb{N} \rightarrow \mathbb{N}$ and all $n \gg 0$.

(1) If $m(n) \sim n^{\beta /(\beta+1)}$ for some real $\beta \geq 0$, then $n^{\alpha} \preccurlyeq f(n)$ for every real number $0<\alpha<\beta+1$.

(2) If $m(n) \geq n-q$ for some $q \in \mathbb{N}$ and all $n \gg 0$, then $\delta^{n} \preccurlyeq f(n)$ for some real $\delta>1$.

Proof. (1) Fix some $\alpha$ with $0<\alpha<\beta+1$. It is a calculus exercise to prove that

$$
\lim _{n \rightarrow \infty} \frac{\left(\frac{n+1}{n}\right)^{\alpha}-1}{\frac{1}{n}}=\alpha,
$$

and so $\left((n+1)^{\alpha}-n^{\alpha}\right) \sim n^{\alpha-1}$. Thus we can find a real $\lambda>0$ with the property that $(n+1)^{\alpha} \leq n^{\alpha}+\lambda n^{\alpha-1}$ for all $n \geq n_{0}$, some $n_{0}>0$. Adjusting $n_{0}$ higher if necessary, we can find a real $\epsilon>0$ such that $m(n) \geq \epsilon n^{\beta /(\beta+1)}$ for all $n \geq n_{0}$. Again adjusting $n_{0}$, since $n$ dominates $n^{\beta /(\beta+1)}$ we can also assume that $n \geq m(n)$ for $n \geq n_{0}$. Finally, the hypothesis $\alpha<\beta+1$ implies that $(\alpha-1)<\alpha \beta /(\beta+1)$ and so with a final enlargement of $n_{0}$ we may also assume that $\epsilon^{\alpha} n^{\alpha \beta /(\beta+1)} \geq \lambda n^{\alpha-1}$ holds for $n \geq n_{0}$.

Since $f(n)$ is positive-valued, we can choose a real $\gamma>0$ so that $f(k) \geq \gamma k^{\alpha}$ holds for all $k \leq n_{0}$. Let $n \geq n_{0}$ and assume we have proven already that $f(k) \geq \gamma k^{\alpha}$ for 
all $k \leq n$. Then

$$
\begin{gathered}
f(n+1) \geq f(n)+f(m(n)) \geq \gamma n^{\alpha}+\gamma m(n)^{\alpha} \geq \gamma n^{\alpha}+\gamma \epsilon^{\alpha} n^{\alpha \beta /(\beta+1)} \\
\geq \gamma n^{\alpha}+\gamma \lambda n^{\alpha-1} \geq \gamma(n+1)^{\alpha} .
\end{gathered}
$$

Thus the inequality $f(n) \geq \gamma n^{\alpha}$ holds for all $n$ by induction.

(2) The linear recurrence relation $g(n+1)=g(n)+g(n-q)$ is well known to give a function $g(n)$ with exponential growth, and the hypothesis in this case implies that $f(n)$ is growing at least this fast.

Next, fix a nonsingular projective curve $C$ over $k$, and let $F=k(C)$ be its field of rational functions. A finite-dimensional subspace $W \subseteq F$ generates an invertible sheaf $\mathcal{M}=W \mathcal{O}_{C}$ on $C$. We will use the following convenient notation in the next result: for any $f \in F$ we let $d(f)=\operatorname{deg} W \mathcal{O}_{C}$ for $W=k+k f$. Alternatively, if $(f)$ is the principal divisor of the rational function $f$ and we write $(f)=P-Q$, where $P$ is the divisor of zeroes and $Q$ is the divisor of poles, then $d(f)=\operatorname{deg} P=\operatorname{deg} Q$ since $(k+k f) \mathcal{O}_{C}=\mathcal{O}_{C}(Q)$. Note that for an arbitrary vector subspace $W \subseteq F$, $\operatorname{deg} W \mathcal{O}_{C}$ gives us no information about $\operatorname{dim}_{k} W$; in fact, every effective invertible sheaf on $C$ can be generated by at most 2 sections. On the other hand, the next lemma shows that if we have a collection of subspaces $V_{0}, V_{1}, V_{2} \cdots \subseteq F$, then knowledge of the growth of $\operatorname{deg} V_{i} \mathcal{O}_{C}$ does allow us to determine the growth of $\operatorname{dim}_{k} V_{0} V_{1} \ldots V_{n-1}$.

Lemma 6.3. Let $C$ be a nonsingular projective curve over $k$ with $F=k(C)$. Let $V_{0}, V_{1}, V_{2}, \cdots \subseteq F$ be a sequence of nonzero finite-dimensional $k$-vector subspaces of $F$, and put $d_{n}=\operatorname{deg} V_{n} \mathcal{O}_{C}$; assume that $d_{n}>0$ for $n \gg 0$. Let $W_{n}=V_{0} V_{1} \ldots V_{n-1}$ for each $n$, and put $e_{n}=\operatorname{dim}_{k} W_{n}$.

(1) Suppose that $d_{n} \sim n^{j}$ for some integer $j \geq 0$. Then for every real number $0<\alpha<j+1$, we have $n^{\alpha} \preccurlyeq e_{n} \preccurlyeq n^{j+1}$.

(2) Suppose that $d_{n} \sim n^{j} \rho^{n}$ for some integer $j \geq 0$ and real $\rho>1$. Then $\delta^{n} \preccurlyeq e_{n}$ for some real $\delta>1$.

Proof. First we give an upper bound on the growth in case (1), so suppose that $d_{n} \sim n^{j}$. Then $\mathcal{M}_{n}=W_{n} \mathcal{O}_{C}$ is an invertible sheaf of degree $h_{n}=\sum_{i=0}^{n-1} d_{i}$ on $C$. Since $d_{n}>0$ for $n \gg 0, h_{n+1}>h_{n}$ for $n \gg 0$. In particular, $h_{n}>2 g-2$ for $n \gg 0$, where $g$ is the genus of $C$. Then $\mathcal{M}_{n}$ is nonspecial for $n \gg 0$ (see [Ha, Example IV.1.3.4]) and so by the Riemann-Roch formula,

$$
e_{n}=\operatorname{dim}_{k} W_{n} \leq \operatorname{dim}_{k} \mathrm{H}^{0}\left(\mathcal{M}_{n}\right)=h_{n}+1-g .
$$

Thus $e_{n} \preccurlyeq h_{n} \sim n^{j+1}$.

Now we work on the lower bounds. Replacing each $V_{i}$ with some $g_{i} V_{i}$ with $0 \neq g_{i} \in F$ does not affect the numbers $d_{i}$ or $e_{i}$, so by making such replacements we may assume that $1 \in V_{i}$ for all $i \geq 0$. Then $W_{n} \subseteq W_{n+1}$ for all $n$. Also, since $\operatorname{dim}_{k} V=1$ implies that $\operatorname{deg} V \mathcal{O}_{C}=0$, for $n \gg 0$ we must have $\operatorname{dim}_{k} V_{n} \geq 2$. Consider some such $n$. There is some finite set of points $S \subseteq C$ such that all elements of $W_{n}=V_{0} V_{1} \ldots V_{n-1}$ have poles only along points of $S$. Since $V_{n}$ is at least 2-dimensional and contains 1 , we may choose a generic $f \in V_{n}$ which does not have zeroes at any point in $S$, and such that $d(f)=d_{n}=\operatorname{deg} V_{n} \mathcal{O}_{C}$.

Given any $g \in W_{n}$, write $(g)=P-Q$, where $P$ is the divisor of zeroes and $Q$ the divisor of poles of $g$. Similarly, write $(f)=P^{\prime}-Q^{\prime}$ and $(f g)=(f)+(g)=P^{\prime \prime}-Q^{\prime \prime}$. By construction, $P^{\prime}$ and $Q$ have disjoint support and so $\operatorname{deg} P^{\prime \prime} \geq \operatorname{deg} P^{\prime}$. We 
conclude that $d(f g) \geq d(f)=d_{n}$ for all $g \in W_{n}$. Now let $m=m(n)$ be the largest integer such that $\operatorname{deg} W_{m} \mathcal{O}_{C}<d_{n}$ (this makes sense, since $\operatorname{deg} W_{m} \mathcal{O}_{C}$ is eventually strictly monotonically increasing by the first paragraph of the proof). Then $d\left(g^{\prime}\right)<d_{n}$ for all $g^{\prime} \in W_{m}$. We conclude that $W_{m} \cap W_{n} f=0$. Since $W_{m}+W_{n} f \subseteq W_{n+1}$, this leads to the formula $e_{n+1} \geq e_{n}+e_{m}$.

Now in case (1), we have $d_{n} \sim n^{j}$ and $\operatorname{deg} W_{n}=h_{n}=\sum_{i=0}^{n-1} d_{i} \sim n^{j+1}$, so it is easy to see that $m(n) \sim n^{j /(j+1)}$. Then for every $0<\alpha<j+1$, we have $n^{\alpha} \preccurlyeq e_{n}$ by Lemma 6.2(1).

In case (2), suppose that $\mu n^{j} \rho^{n} \leq d_{n} \leq \epsilon n^{j} \rho^{n}$ for all $n \gg 0$ and some $0<\mu<\epsilon$. Note that

$$
\operatorname{deg} W_{n} \mathcal{O}_{C}=\sum_{i=0}^{n-1} d_{i} \leq \epsilon \sum_{i=0}^{n-1} i^{j} \rho^{i} \leq \epsilon n^{j}\left(\rho^{n}-1\right) /(\rho-1) .
$$

Now it is easy to see that there is an integer $q>0$ such that for all $n \geq 0$, and for any $0 \leq \ell \leq n-q$, we have $\epsilon \ell^{j}\left(\rho^{\ell}-1\right) /(\rho-1)<\mu n^{j} \rho^{n}$. Thus $m(n) \geq n-q$, and so $\delta^{n} \preccurlyeq e_{n}$ for some real $\delta>1$ by Lemma $6.2(2)$.

Now we are ready to give a lower bound on the growth of certain subalgebras of $\widetilde{B}(X, \mathcal{L}, \sigma)$.

Proposition 6.4. Assume Notation [5.1, so $\sigma: X \rightarrow X$ is a stable birational map of a nonsingular projective model $X$ of $K$, with corresponding growth data $(\rho, j)$. Assume that the base field $k$ is uncountable. By Lemma 4.4, pick a very ample invertible sheaf $\mathcal{L} \subseteq \mathcal{K}$ which is $\sigma$-positive, and let $U=\mathrm{H}^{0}(X, \mathcal{L}) \subseteq K$. Let

$$
A=k\langle U t\rangle \subseteq \widetilde{B}(X, \mathcal{L}, \sigma)=\bigoplus_{n \geq 0} \mathrm{H}^{0}\left(X, \mathcal{L}_{n}\right) t^{n} \subseteq Q=K\left[t, t^{-1} ; \sigma\right] .
$$

Then $A$ has exponential growth if $\rho>1$, and GK $A \geq j+3$ if $\rho=1$.

Proof. For each $n \in \mathbb{Z}$ the birational map $\sigma^{n}: X \rightarrow X$ is defined except at a finite set of points $S_{n}$ of $X$. We may adjust the embedding $\mathcal{L} \subseteq \mathcal{K}$ if necessary so that $1 \in U=\mathrm{H}^{0}(X, \mathcal{L}) \subseteq K$. Let $\mathcal{L}=\mathcal{O}_{X}(D)$, and consider the complete linear system $|D|$ on $X$. Using Bertini's Theorem and the fact that $k$ is uncountable, we may choose a Weil divisor $E \in|D|$ with the following properties: (i) $E$ is an irreducible nonsingular curve, and (ii) $E$ contains none of the countably many points in $S=\bigcup_{n \geq 1} S_{n}$.

Let $\mathcal{I}=\mathcal{O}_{X}(-E)$ be the ideal sheaf of the divisor $E$. It follows from the definition of $\widetilde{B}=\widetilde{B}(X, \mathcal{L}, \sigma)$ that $I=\bigoplus_{n \geq 0} \mathrm{H}^{0}\left(X, \mathcal{I} \otimes \mathcal{L}_{n}\right) t^{n}$ is a homogeneous right ideal of $\widetilde{B}$. Recalling the notational convention $U^{\sigma}=\sigma(U)$ from Section 5 , write $U_{n}=U U^{\sigma} \ldots U^{\sigma^{n-1}} \subseteq K$ for each $n \geq 1$ and $U_{0}=k$, so that $A=\bigoplus_{n \geq 0} U_{n} t^{n}$. For each $i$, the vector space $U^{\sigma^{i}}$ generates the sheaf $\mathcal{L}^{\sigma^{i}}$ except possibly at points in $S_{i} \subseteq S$, by Lemma 4.1. Put $\mathcal{M}^{(i)}=\mathcal{L}^{\sigma^{i}}{ }_{E}$ and let $V_{i} \subseteq \mathrm{H}^{0}\left(E, \mathcal{M}^{(i)}\right)$ be the image of $U^{\sigma^{i}} \subseteq \mathrm{H}^{0}\left(X, \mathcal{L}^{\sigma^{i}}\right)$ under the restriction of sections map. By the choice of $E, \mathcal{M}^{(i)}$ is an invertible sheaf on $E$ which is generated by the sections in $V_{i}$ everywhere. Similarly, put $\mathcal{M}_{n}=\left.\mathcal{L}_{n}\right|_{E}$ and let $W_{n} \subseteq \mathrm{H}^{0}\left(E, \mathcal{M}_{n}\right)$ be the image of $U_{n} \subseteq \mathrm{H}^{0}\left(X, \mathcal{L}_{n}\right)$ under the restriction of sections map; again, $W_{n}$ generates $\mathcal{M}_{n}$ on $E$. Choose arbitrary embeddings $\mathcal{M}^{(i)} \subseteq \mathcal{F}$ for each $i$, where $\mathcal{F}$ is the constant sheaf on $F=k(E)$, which determines an embedding of $\mathcal{M}_{n}=\mathcal{M}^{(0)} \otimes \mathcal{M}^{(1)} \otimes \cdots \otimes \mathcal{M}^{(n-1)}$ in $\mathcal{F}$ for each $n$. This also embeds $V_{i} \subseteq F$ for each $i$ and $W_{n} \subseteq F$ for each $n$, and 
since $U_{n}=U U^{\sigma} \cdots U^{\sigma^{n-1}}$ in $K$, we have $W_{n}=V_{0} V_{1} \cdots V_{n-1}$ in $F$. Observe that there is a graded vector space map $\theta: A \rightarrow \bigoplus_{n>0} \mathrm{H}^{0}\left(E, \mathcal{M}_{n}\right)$ given by restriction of sections, with kernel $A \cap I=\bigoplus_{n \geq 0}\left(U_{n} \cap \mathrm{H}^{0}\left(X, \mathcal{I} \otimes \mathcal{L}_{n}\right)\right) t^{n}$ and image $\bigoplus_{n \geq 0} W_{n}$. (In fact, $\bigoplus_{n \geq 0} \mathrm{H}^{0}\left(E, \mathcal{M}_{n}\right)$ is naturally a graded right $A$-module and $\theta$ is a right module map, but we won't need this.)

In case $\rho>1$, to show that $A$ has exponential growth it will clearly suffice to show that $\operatorname{dim}_{k} W_{n}$ grows exponentially with $n$. In case $\rho=1$, note that by construction, $A_{1}=\mathrm{H}^{0}(X, \mathcal{L}) t$ and $\mathcal{L}$ contains a nonzero section vanishing along the curve $E$, so $(A \cap I)_{1} \neq 0$. In particular, the GK-dimension of the right $A$-module $A /(A \cap I)$ satisfies GK $A /(A \cap I) \leq$ GK $A-1$ by [KL, Proposition 5.1(e)]. Then if we can show that $n^{\alpha} \preccurlyeq \operatorname{dim}_{k} W_{n}$ holds for all $\alpha<j+1$, we will have $j+2 \leq \operatorname{GK} A /(A \cap I)$ by [KL, Lemma $6.1(\mathrm{~b})]$, and so $j+3 \leq \mathrm{GK} A$.

Now on the curve $E$, putting $d_{n}=\operatorname{deg} V_{n} \mathcal{O}_{E}=\operatorname{deg} \mathcal{M}^{(n)}$ we see that $d_{n}=$ $\left(\left(\sigma^{n}\right)^{*}(E) . E\right)$ by [Ha, Lemma V.1.3]. Then since $E$ is ample, $d_{n} \sim n^{j} \rho^{n}$ by Lemma 5.4. In particular, notice that $d_{n}>0$ for all $n \geq 0$. Finally, by Lemma 6.3. putting $e_{n}=\operatorname{dim}_{k} W_{n}$, then $e_{n}$ has exponential growth if $\rho>1$, while $n^{\alpha} \preccurlyeq e_{n}$ for all $\alpha<j+1$ if $\rho=1$. The result follows.

\section{Proof of the MAIN THEOREM}

We now put together the various estimates already proved to calculate the GKdimension of big subalgebras $A \subseteq K\left[t, t^{-1} ; \sigma\right]$.

Theorem 7.1. Let $k$ be an uncountable algebraically closed field. Define $Q=$ $K\left[t, t^{-1} ; \sigma\right]$, where $K$ is a finitely generated field extension of $k$ with $\operatorname{tr} . \operatorname{deg} K / k=2$ and $\sigma \in \operatorname{Aut}_{k}(K)$. Let $(\rho, j)$ be the growth data associated to $\sigma$, as in Notation 5.1 . Given any big locally finite $\mathbb{N}$-graded subalgebra $A \subseteq Q$, then GK $A$ is determined as follows:

(1) If $\rho>1$, then $A$ has exponential growth (in particular, GK $A=\infty$ ).

(2) If $\rho=1$, then GK $A=j+3$. In particular, GK $A \in\{3,4,5\}$ and GK $A=4$ if and only if $\sigma$ is nongeometric.

Proof. Suppose first that $\rho=1$. Choose a nonsingular projective model $X$ of $K$ so that the birational map $\sigma: X \rightarrow X$ satisfies Hypothesis 5.2. Write $A=$ $\bigoplus_{n \geq 0} U_{n} t^{n}$, where $U_{n} \subseteq K$. Assume for the moment that $A$ is finitely generated as an algebra. Since $A_{0}$ is a finite-dimensional $k$-algebra which is a domain and $k$ is algebraically closed, $A_{0}=k$. Suppose that $A$ is generated by elements of degree $\leq d$. Letting $W=k+U_{1}+\cdots+U_{d}$, then $A$ is contained in the $\operatorname{ring} k\langle W t\rangle \subseteq Q$. Moreover, we can find a very ample invertible sheaf $\mathcal{L}$ with an embedding $\mathcal{L} \subseteq \mathcal{K}$ in the constant sheaf of rational functions such that $W \subseteq \mathrm{H}^{0}(X, \mathcal{L}) \subseteq K$ [RZ, Lemma 5.2]. Replace $\mathcal{L}$ by a power $\mathcal{L}^{\otimes m}$ if necessary, so that $\mathcal{L}$ is $\sigma$-positive, using Lemma 4.4. Then $k\langle W t\rangle \subseteq \bigoplus_{n \geq 0} \mathrm{H}^{0}\left(X, \mathcal{L}_{n}\right) t^{n}=\widetilde{B}(X, \mathcal{L}, \sigma) \subseteq Q$. But in Proposition 5.6 we saw that $\operatorname{dim}_{k} \widetilde{B}_{n} \sim n^{j+2}$; hence GK $A \leq j+3$. Now since this estimate holds for all finitely generated $A$, GK $A \leq j+3$ holds for an arbitrary locally finite $\mathbb{N}$-graded subalgebra $A$ of $Q$, by definition.

Now we find a lower bound for GK $A$. Choose any invertible sheaf $\mathcal{L} \subseteq \mathcal{K}$ which is very ample and $\sigma$-positive and let $W=\mathrm{H}^{0}(X, \mathcal{L}) \subseteq K$. Suppose first that $\rho>1$, so we want to prove that $A$ has exponential growth. In this case, by [RZ, Proposition 1.4] it suffices to find any finitely generated $\mathbb{N}$-graded subalgebra of $Q$ 
with exponential growth, and so we will prove that $k\langle W t\rangle \subseteq Q$ has exponential growth.

If instead $\rho=1$, then the first part of the proof shows that GK $A<\infty$, so $A$ is an Ore domain by [KL, Proposition 4.13]. Then again setting $A=\bigoplus_{n \geq 0} U_{n} t^{n}$, the same argument as in [RZ, Lemma 5.3] (using that $A$ is big in $Q$ ) shows that there is some $n>0$ and $z \in U_{n}$ such that $W z \subseteq U_{n}$. Setting $t^{\prime}=t^{n}$ and $\sigma^{\prime}=\sigma^{n}$, the Veronese ring $A^{\prime}=A^{(n)}$ contains $k\left\langle W z t^{\prime}\right\rangle$, which is a big subalgebra of $Q^{\prime}=Q^{(n)}=K\left[t^{\prime},\left(t^{\prime}\right)^{-1} ; \sigma^{\prime}\right]$. Note that the induced map $\sigma^{\prime}=\sigma^{n}: X \rightarrow X$ is again a stable birational map, with the same associated growth data $(\rho, j)=(1, j)$. Moreover, $k\left\langle W z t^{\prime}\right\rangle \cong k\left\langle W t^{\prime}\right\rangle$. It is enough to put a lower bound on GK $A^{\prime}$, so we now change notation back by removing the primes, and our task is again to find a lower bound for the GK-dimension of $k\langle W t\rangle \subseteq Q$.

Thus in both cases for $\rho$, the rings $k\langle W t\rangle \subseteq \bigoplus_{n \geq 0} \mathrm{H}^{0}\left(X, \mathcal{L}_{n}\right) t^{n}=\widetilde{B}(X, \mathcal{L}, \sigma)$ now satisfy the hypothesis of Proposition 6.4. By that proposition, $k\langle W t\rangle$ has exponential growth if $\rho>1$ and GK $k\langle W t\rangle \geq j+3$ if $\rho=1$.

Taking the upper and lower bounds together, the calculation of GK $A$ is complete. The rest of part (2) follows immediately from the classification in Theorem 3.2 .

Proof of Theorem 1.1. The theorem follows immediately from Theorem 7.1 if $k$ is uncountable, so we just need to reduce to this case. Suppose that $k$ is any algebraically closed field. Let $A \subseteq K\left[t, t^{-1} ; \sigma\right]$ be a big locally finite $\mathbb{N}$-graded subalgebra, where $K / k$ is a finitely generated field extension with tr. $\operatorname{deg} K / k=2$. Choose any field extension $k \subseteq L$, where $L$ is uncountable and algebraically closed. As was also noted in Lemma 3.7, since $k$ is algebraically closed, $K \otimes_{k} L$ is again a commutative domain; letting $F$ be its field of fractions, $\sigma$ extends uniquely to an automorphism $\widetilde{\sigma} \in \operatorname{Aut}_{L} F$. Consider $\widetilde{A}=A \otimes_{k} L \subseteq K\left[t, t^{-1} ; \sigma\right] \otimes_{k} L \subseteq F\left[t, t^{-1} ; \widetilde{\sigma}\right]$. It is easy to see that $\widetilde{A}$ is a big finitely $\mathbb{N}$-graded subalgebra of $F\left[t, t^{-1} ; \widetilde{\sigma}\right]$, where again $F / L$ is a finitely generated field extension with tr. $\operatorname{deg} F / L=2$. Also, $\mathrm{GK}_{L} \widetilde{A}=\mathrm{GK}_{k} A$. Now the result follows from Theorem 7.1 , together with Lemma 3.7

\section{ACKNOWLEDGMENTS}

This paper benefited from conversations with James Zhang, Mike Artin, and Toby Stafford, and we thank them.

\section{REFERENCES}

[AS] M. Artin and J. T. Stafford, Noncommutative graded domains with quadratic growth, Invent. Math. 122 (1995), no. 2, 231-276. MR96g:16027

[AV] M. Artin and M. Van den Bergh, Twisted homogeneous coordinate rings, J. Algebra 133 (1990), no. 2, 249-271. MR.91k:14003

[BPV] W. Barth, C. Peters, and A. Van de Ven, Compact complex surfaces, Springer-Verlag, Berlin, 1984. MR.749574 (86c:32026)

[DF] J. Diller and C. Favre, Dynamics of bimeromorphic maps of surfaces, Amer. J. Math. 123 (2001), no. 6, 1135-1169. MR2002k:32028

[Fj] Takao Fujita, Vanishing theorems for semipositive line bundles, Algebraic geometry (Tokyo/Kyoto, 1982), Lecture Notes in Math., vol. 1016, Springer, Berlin, 1983, pp. 519528. MR $85 \mathrm{~g}: 14023$

[Ha] Robin Hartshorne, Algebraic geometry, Springer-Verlag, New York, 1977, Graduate Texts in Mathematics, No. 52. MR57:3116

[Ke1] D. S. Keeler, Criteria for $\sigma$-ampleness, J. Amer. Math. Soc. 13 (2000), no. 3, 517-532. MR2001d:14003

[Ke2] D. S. Keeler, Ample filters and Frobenius amplitude, preprint, arXiv:math/0603388. 
[KL] Günter R. Krause and Thomas H. Lenagan, Growth of algebras and GelfandKirillov dimension, revised ed., American Mathematical Society, Providence, RI, 2000. MR2000j:16035

[KRS] D. S. Keeler, D. Rogalski, and J. T. Stafford, Nä̈ve noncommutative blowing up, Duke Math. J. 126 (2005), no. 3, 491-546. MR2120116

[La] Robert Lazarsfeld, Positivity in algebraic geometry I, Springer-Verlag, Berlin, 2004. MR.2095471 (2005k:14001a)

[RS1] D. Rogalski and J. T. Stafford, A class of noncommutative projective surfaces, preprint, arXiv:math/0612657.

[RS2] D. Rogalski and J. T. Stafford, Nä̈ve noncommutative blowups at zero-dimensional schemes, J. Algebra 318 (2007), no. 2, 794-833. MR2371973

[RZ] D. Rogalski and J. J. Zhang, Canonical maps to twisted rings, Math. Z. 259 (2008), no. 2, 433-455. MR2390090

[Sh] Igor R. Shafarevich, Basic algebraic geometry I, second ed., Springer-Verlag, Berlin, 1994. MR.1328833 (95m:14001)

[V] James S. Vandergraft, Spectral properties of matrices which have invariant cones, SIAM J. Appl. Math. 16 (1968), 1208-1222. MR0244284 (39:5599)

Department of Mathematics, University of California, San Diego, La Jolla, CaliFORNIA 92093-0112

E-mail address: drogalsk@math.ucsd.edu 